\title{
Outage Probability in the Uplink of Multitier Millimeter Wave Cellular Networks
}

\author{
Oluwakayode Onireti (i), Member, IEEE, Lei Zhang (i), Member, IEEE, Ali Imran, Senior Member, IEEE, \\ and Muhammad Ali Imran, Senior Member, IEEE
}

\begin{abstract}
In this article, using the stochastic geometry, we develop a tractable uplink modeling framework for the outage probability of the multitier millimeter wave (mmWave) cellular networks. Each tier's mmWave base stations (BSs) are randomly located and they have particular spatial density, antenna gain, receiver sensitivity, blockage parameter, and pathloss exponents. Our model takes account of the maximum power limitation and the per-user power control. More specifically, each user, which could be in line-of-sight (LOS) or non-LOS to its serving mmWave BS, controls its transmit power such that the received signal power at its serving BS is equal to a predefined threshold. Hence, a truncated channel inversion power control scheme is implemented for the uplink of mmWave cellular networks. We derive closed-form expressions for the signal-to-interference-plus-noise-ratio (SINR) outage probability for the uplink of the multitier mmWave cellular networks, which we later degrade to the single-tier network. Furthermore, we analyze the case with a dense network by utilizing the simplified model, where the LOS region is approximated as a fixed LOS disk. The results show that imposing a maximum power constraint on the user significantly affects the SINR outage probability in the uplink of mmWave cellular networks.
\end{abstract}

Index Terms-Millimeter wave (mmWave), power control, stochastic geometry, truncated channel inversion, uplink communication.

\section{INTRODUCTION}

A FUNDAMENTAL requirement for the 5G-and-beyond mobile networks is the radical increase in data rate. Recent studies have identified massive multiple-input-multiple-output (MIMO), extreme network densification, and increased bandwidth as the key technologies toward meeting this requirement [1]. The millimeter wave (mmWave) frequencies (ranging from $30-300 \mathrm{GHz}$ ) offers a large available bandwidth thus, making them attractive for the 5G mobile networks [1]-[3]. Meanwhile, the mmWave band has long been considered illsuited for the cellular communication due to the excessive pathloss and the poor penetration through materials such as

Manuscript received February 19, 2019; revised December 18, 2019; accepted December 25, 2019. Date of publication January 27, 2020; date of current version June 3, 2020. This work was supported in part by the Engineering and Physical Sciences Research Council U.K. under Grant EP/S02476X/1 and Grant EP/P028764/1, and in part by the National Science Foundation under Grant 1730650 and Grant 1559483. (Corresponding author: Oluwakayode Onireti.)

O. Onireti, L. Zhang, and M. A. Imran are with the James Watt School of Engineering, University of Glasgow, Glasgow G12 8QQ, U.K. (e-mail: oluwakayode.onireti@glasgow.ac.uk; lei.zhang@glasgow.ac.uk; muhammad. imran@glasgow.ac.uk).

A. Imran is with the School of Electrical and Computer Engineering, University of Oklahoma, Tulsa, OK 74135 USA (e-mail: ali.imran@ou.edu).

Digital Object Identifier 10.1109/JSYST.2020.2965001 concrete and water. Recent capacity studies and survey measurement on mmWave technologies in [3]-[6] have shown its great promise for $5 \mathrm{G}$ urban small cell deployments. The recent advances in low-power CMOS RF circuit and the smaller wavelength associated with the band have further substantiated this promise. The later also makes it viable to have more miniaturized antennas within the same physical area of the transmitter and receiver [2], [6]. Furthermore, with a large antenna array, the mmWave network can apply beamforming at the transmit and receive sides to provide array gain that compensates for the pathloss [7]. The directionality gained from beamforming will lead to a reduction in interference [5]. Hence, a mmWave spectrum holds great potential for providing the high data rate (Gigabits range) expected in the upcoming $5 \mathrm{G}$ cellular networks [8].

Modeling and analysis of cellular networks by using stochastic geometry have recently received significant attention due to its high accuracy and tractability. In this approach, the network topology is abstracted to a point process for ease of modeling and analysis. Earlier works in this area were mainly focused on the conventional ultrahigh-frequency (UHF) cellular networks [9][19]. In the pioneering work on using the stochastic geometry for cellular networks [9], it was shown that the stochastic model provides a lower bound to real cellular deployment. The work in [9] was based on the downlink of cellular networks with the single-slope pathloss model. This has been extended by considering a multislope pathloss model in [10] and [11], the multitier cellular networks in [12]-[14], the single-tier uplink cellular networks in [15], and the multitier uplink cellular networks in [16]-[18].

The stochastic geometry framework developed for the UHF networks do not directly apply to the mmWave networks due to blockage effects that they suffer from and the much different pathloss model. Furthermore, directional beamforming is fundamental in the design of the mmWave cellular networks. The authors in [8] and [20]-[24] have analyzed the mmWave cellular networks by using the stochastic geometry framework with the blockage effect, realistic pathloss model and the beamforming gain incorporated in their model. In particular, [8] pioneered the research work on the downlink of mmWave cellular networks by leveraging on an earlier work in [25], which characterized the blockage parameter by some random distribution. Furthermore, the proposed analytical framework in [8] also captures the significant difference between the non-line-of-sight (NLOS) and line-of-sight (LOS) pathloss characteristics. The work in [8] has been extended to the downlink multitier mmWave cellular networks in [20], base station (BS) cooperation in [21] and the uplink single-tier mmWave cellular networks in [22]-[24].

The uplink analysis for both the conventional UHF cellular networks and the mmWave cellular networks are deemed to 
be quite involved as compared to the downlink analysis due to the per-user power control and the correlation among the interferers [16]. The former is due to the fact that an interfering user could even be closer to a reference mmWave BS than the user that is tagged to the reference mmWave BS. Furthermore, regarding mmWave cellular networks, measurements showed that mmWave signals propagate with a pathloss exponent of 2 in LOS paths and a much higher pathloss exponent with additional shadowing in NLOS paths [3], [4]. This poses a further challenge since the difference in pathloss exponents could results in excessive interference from NLOS users when the per-user power control is implemented. Hence, power control must be implemented with a constraint on the maximum user transmit power in order to mitigate the interference. On the other hand, the correlation among the interferer results from the implementation of the orthogonal allocation scheme that does not allow for a reuse of a channel resource within the same cell, i.e., the coupling of the mmWave BS and served user-per channel point processes [15], [16]. For analytical tractability, various generative models have been proposed in [15]-[17] and [22]-[24] to approximate the spatial distribution of interferers in the uplink of UHF and mmWave cellular networks.

In this article, we present a stochastic geometry framework for modeling and analyzing the uplink of a multitier mmWave cellular network. Similar to the earlier works in this area [15]-[17], [22]-[24], we rely on some approximation so as to maintain analytical tractability. Notably, we partially ignore the correlation among the interfering users. Our model captures the correlation between the interfering users and the reference mmWave BS, which serves the typical user, but it ignores the correlation among the interfering users. As evidence from [15]-[17], this approximation holds true for the uplink of both the single- and multi-tier UHF cellular networks. The accuracy of this approach is validated via Monte Carlo simulations. We here extend the work in [24], which is based on a single-tier mmWave network and does not take into account the maximum transmit power of the user. Our proposed framework takes into account the limitation in the user transmit power, the per-user power control and the cutoff threshold for the power control. We compare the findings of our analysis with that from [24]. The comparison reveals that our analysis provides several new insights that can be leveraged for designing the mmWave networks more accurately. The main contributions of this article are summarized as follows.

1) We present a stochastic geometry framework for the signal-to-interference-plus-noise-ratio (SINR) outage probability in the uplink of the multitier mmWave cellular networks. The model takes into account the limitation in the transmit power of the user, and the network-defined per-user power control and the cutoff threshold. The motivation for the multitier model comes from the fact that the single-tier model fails to capture the realistic network deployment that include varied blockage size and intensity, varied BS beamforming gain, varied receiver sensitivity, and multiple dominant LOS and NLOS pathloss exponents over the entire network. We derive a closed-form expression for the SINR outage probability in multitier mmWave networks.

2) We present the asymptotic dense network analysis of the SINR outage probability in multitier mmWave cellular networks. The asymptotic analysis leverage on approximating an intricate LOS function as a step function.

3) The analytical derivations are verified via Monte Carlobased simulations. Results show that the maximum power constraint significantly affects the SINR outage probability. Furthermore, contrary to the SINR outage of UHF networks, which is nonincreasing in the cutoff threshold, the SINR outage probability in mmWave networks could increase over some range of cutoff thresholds for some mmWave BS density, LOS and NLOS pathloss exponents, and blockage parameter.

The rest of this article is organized as follows. The system model of the uplink of a multitier mmWave cellular network with truncated channel inversion power control is presented in Section II. In Section III, the uplink modeling framework for the multitier mmWave cellular network is presented. In Section IV, we utilize a simplified system model to analyze the asymptotic behavior and performance in dense mmWave networks. Numerical and simulation results are presented in Section V. Finally, Section VI concludes this article. A preliminary version of this work has been reported in [26]. Herein, we have presented the distribution of the transmit power and the SINR outage probability in the uplink of a multitier mmWave cellular network with truncated power control. Furthermore, the asymptotic dense network analysis have been derived for the multitier mmWave cellular networks.

\section{SYSTEM MODEL}

\section{A. Network Model}

We consider the uplink of a $K$-tier mmWave cellular network and focus on the SINR experienced by outdoor users served by outdoor mmWave BSs. Each tier's BSs are randomly located and they have particular spatial density, antenna gain, receiver sensitivity, blockage parameter, and pathloss exponents. The outdoor BSs of each tier are spatially distributed in $\mathbb{R}^{2}$ according to an independent homogeneous Poisson point process (PPP) $\Theta_{k}$ with density $\lambda_{k}$. The users locations (before association) are assumed to form a realization of homogeneous PPP $\Phi$ with density $\lambda_{u}$. It is assumed that the density of the users is high enough such that each BS will have at least one user. Each BS serves a single user, which is randomly selected from all the users located in its Voronoi cell by using a round-robin scheduler. As in [15]-[17], [19], and [22]-[24], we assume that the active users also form PPP even after associating just one user per BS. Note that this approximation only partly ignores the correlation imposed by the system model, i.e., the coupling of the BS and served user point processes. The correlation between the reference mmWave BS and the typical user is captured in the derivation of the outage probability in Sections III and IV.

Similar to [21], each tier in the mmWave network is characterized by a nonnegative blockage constant $\beta_{k}$ for $k \in\{1, \ldots, K\}$. The parameter $\beta_{k}$ is determined by the average size and density of blockages in that tier and where the average LOS range is given by $\frac{1}{\beta_{k}}[8],[21],[25]$. The probability of a communication link in the $k$ th tier with length $r$ being an LOS is $\mathbb{P}\left(\operatorname{LOS}_{k}\right)=e^{-\beta_{k} r}$, while the probability of a link being NLOS is $\mathbb{P}\left(\mathrm{NLOS}_{k}\right)=1-\mathbb{P}\left(\operatorname{LOS}_{k}\right)$. The LOS and NLOS links of the $k$ th tier have different pathloss exponents denoted by $\alpha_{L}^{k}$ and $\alpha_{N}^{k}$, respectively, $\forall k \in\{1, \ldots, K\}$.

\section{B. Receiver Sensitivity and Truncated Outage}

We assume that all users have an equal maximum transmit power $P_{u}$. Furthermore, all mmWave BS in the $k$ th tier 
TABLE I

PROBABILITY MASS FUNCTION OF THE DIRECTIVITY GAIN IN AN INTERFERENCE LINK OF THE $j$ th TIER [25]

\begin{tabular}{c|c|c|c|c}
\hline$v$ & 1 & 2 & 3 & 4 \\
\hline$a_{v}^{j}$ & $G_{b j}^{\max } G_{u}^{\max }$ & $G_{b j}^{\max } G_{u}^{\min }$ & $G_{b j}^{\min } G_{u}^{\max }$ & $G_{b j}^{\min } G_{u}^{\min }$ \\
\hline$b_{v}^{j}$ & $\frac{\zeta_{r j} \zeta_{t}}{4 \pi^{2}}$ & $\frac{\zeta_{r j}}{2 \pi}\left(1-\frac{\zeta_{t}}{2 \pi}\right)$ & $\left(1-\frac{\zeta_{r j}}{2 \pi}\right) \frac{\zeta_{t}}{2 \pi}$ & $\left(1-\frac{\zeta_{r j}}{2 \pi}\right)\left(1-\frac{\zeta_{t}}{2 \pi}\right)$ \\
\hline
\end{tabular}

have the same receiver sensitivity, which is denoted by $\rho_{\min }^{k}$. The received signal at the mmWave BS must be greater than the receiver sensitivity $\rho_{\min }^{k}$ for successful transmission in the uplink channel. Hence, each user (with either LOS or NLOS link to its serving mmWave BS) associated with the $k$ th tier adjusts its transmit power such that the average received signal at its serving mmWave BS is equal to a predefined threshold $\rho_{o}^{k}$, where $\rho_{o}^{k}>\rho_{\min }^{k}$. Moreover, as a result of the maximum transmit power constraint, users utilize a truncated channel inversion power control scheme, where the transmitters compensate for the pathloss in the link to the receiver to keep the average received signal power to the threshold $\rho_{o}^{k}$. Any user-mmWave BS connection that requires a transmit power that exceeds $P_{u}$ for the pathloss inversion will not be established, hence, such a connection experiences a truncation outage [16].

\section{Beamforming Gain}

For analytical tractability, we assume that all users and BSs are equipped with directional antennas with a sectorized gain pattern. The main lobe gain, side lobe gain, and beamwidth of the users are $G_{u}^{\max }, G_{u}^{\min }$, and $\zeta_{t}$, respectively, while the corresponding parameters of the $k$ th tier BS antennas are $G_{b k}^{\max }$, $G_{b k}^{\min }$, and $\zeta_{r k}$, respectively. We consider that based on channel estimation, the reference BS in the $j$ th tier and the typical user adjust their beam steering angles to achieve the maximum array gains. As a result, the total directivity gain of the desired signal is $\mathcal{G}_{j}=G_{b j}^{\max } G_{u}^{\max }$. Since the underlying PPP is isotropic in $\mathbb{R}^{2}$, we model the beam directions of the interfering link as a uniform random variable on $[0,2 \pi]$. Furthermore, the directivity gain in the interference link $G_{l}^{j}$ (interference experienced at the reference $\mathrm{BS}$ in the $j$ th tier) can be approximated as discrete random variable whose probability distribution is given as $a_{v}^{j}$ with probability $b_{v}^{j}(v \in\{1,2,3,4\})[25]$, where $a_{v}^{j}$ and $b_{v}^{j}$ are defined in Table I.

In general, the $k$ th tier is characterized by a set of parameters $\mathcal{V}_{k}$ whose element include the $k$ th tier's BS density $\lambda_{k}$, blockage parameter $\beta_{k}$, cutoff threshold $\rho_{o}^{k}$, main lobe gain $G_{b k}^{\max }$, side lobe gain $G_{b k}^{\min }$, beamwidth $\zeta_{r k}$, LOS pathloss exponent $\alpha_{L}^{k}$, and its NLOS pathloss exponent $\alpha_{N}^{k}$ such that $\mathcal{V}_{k}=\left\{\lambda_{k}, \beta_{k}, \rho_{o}^{k}, G_{b k}^{\max }, G_{b k}^{\min }, \zeta_{r k}, \alpha_{L}^{k}, \alpha_{N}^{k}\right\} \forall k=1, \ldots, K$.

\section{Uplink OF MUltitier MMWAVE CEllular Networks}

In this section, we develop our framework model for the uplink of a multitier mmWave cellular network. In particular, we present the mmWave transmission power analysis and the SINR outage probability analysis for the multitier network, which we later degrade to the single-tier network.

\section{A. Distribution of the Transmit Power in the Uplink of Multitier mmWave Cellular Networks}

Considering the mmWave cellular network with the truncated channel inversion scheme, each user, which could be in LOS or
NLOS to its serving mmWave BS will transmit with different power in order to invert the pathloss toward its serving BS. As a result of the truncation channel inversion, not all users will be able to communicate in the uplink channel. In particular, given the cutoff threshold for the $k$ th tier $\rho_{o}^{k}$, LOS and NLOS users located at distances greater than $\left(P_{u} / \rho_{o}^{k}\right)^{1 / \alpha_{L}^{k}}$ and $\left(P_{u} / \rho_{o}^{k}\right)^{1 / \alpha_{N}^{k}}$, respectively, from their associated mmWave $\mathrm{BS}$ are unable to communicate in the uplink direction due to insufficient transmit power. Hence, in addition to the fact that the whole user set is divided into a subset of LOS and NLOS users based on their association with their serving mmWave BS, the LOS and NLOS user sets in the $k$ th tier are further divided into a nonoverlapping subset of active users and inactive users. The distribution of the transmit power of a typical user associated with the $j$ th tier is obtained from the following theorem.

Theorem III.1: In a $K$-tier mmWave cellular network with truncated channel inversion power control where the $k$ th tier is distinguished by the set $\mathcal{V}_{k} \forall k=1, \ldots, K$, i.e., its density $\lambda_{k}$, blockage parameter $\beta_{k}$, cutoff threshold $\rho_{o}^{k}$, antenna parameters, $G_{b k}^{\max }, G_{b k}^{\min }$, and $\zeta_{r k}$, LOS pathloss exponent $\alpha_{L}^{k}$ and its NLOS pathloss exponent $\alpha_{N}^{k}$, the PDF of the transmit power of a typical active user in the uplink of the $j$ th tier is given by

$$
f_{P_{j}}(p)=\frac{\sum_{k=1}^{K} \bar{\lambda}_{k}(p)}{1-e^{-\sum_{a=1}^{K} \Lambda_{a}\left(\frac{P_{u}}{\rho_{o}^{j}}\right)}} e^{-\sum_{b=1}^{K} \Lambda_{b}\left(\frac{p}{\rho_{o}^{j}}\right)}
$$

where

$$
\begin{aligned}
\bar{\lambda}_{k}(p)= & \frac{2 \pi \lambda_{k}}{\alpha_{L}^{k} \rho_{o}^{j / \alpha_{L}^{k}}} p^{\frac{2}{\alpha_{L}^{k}}-1} e^{-\beta_{k}\left(\frac{p}{\rho_{o}^{j}}\right)^{\frac{1}{\alpha_{L}^{k}}}} \\
& +\frac{2 \pi \lambda_{k}}{\alpha_{N}^{k} \rho_{o}^{j 2 / \alpha_{N}^{k}}} p^{\frac{2}{\alpha_{N}^{k}}-1}\left(1-e^{-\beta_{k}\left(\frac{p}{\rho_{o}^{j}}\right)^{\frac{1}{\alpha_{k}^{k}}}}\right) \\
\Lambda_{k}(y)= & \frac{2 \pi \lambda_{k}}{\beta_{k}^{2}}\left(1-e^{-\beta_{k} y^{\frac{1}{\alpha_{L}^{k}}}}\left(1+\beta_{k} y^{\frac{1}{\alpha_{L}^{k}}}\right)\right)+\pi \lambda_{k} y^{\frac{2}{\alpha_{N}^{k}}} \\
& -\frac{2 \pi \lambda_{k}}{\beta_{k}^{2}}\left(1-e^{\left.-\beta_{k} y^{\frac{1}{\alpha_{N}^{k}}}\left(1+\beta_{k} y^{\frac{1}{\alpha_{N}^{k}}}\right)\right)}\right.
\end{aligned}
$$

and $y$ is a dummy variable in (3).

Proof: See Appendix A

The $\delta$ th moment of the transmit power of a user in the $j$ th tier is given as

$$
\mathbb{E}\left[P_{j}^{\delta}\right]=\int_{0}^{P_{u}} \frac{p^{\delta} \sum_{k=1}^{K} \bar{\lambda}_{k}(p)}{1-e^{-\sum_{a=1}^{K} \Lambda_{a}\left(\frac{P_{u}}{\rho_{o}^{j}}\right)}} e^{-\sum_{b=1}^{K} \Lambda_{b}\left(\frac{p}{\rho_{o}^{j}}\right)} \mathrm{d} p .
$$

Furthermore, the truncation outage probability in the uplink of mmWave cellular networks for the $j$ th tier can be obtained as

$$
\mathcal{O}_{p}^{j}=e^{-\sum_{k=1}^{K} \Lambda_{k}\left(\frac{P_{u}}{\rho_{o}^{j}}\right)} .
$$

For a single-tier mmWave network, ${ }^{1}$ the distribution of the transmit power of a typical user is obtained from the following corollary.

\footnotetext{
${ }^{1}$ Note that for all the parameters in the single-tier network, we have removed the subscript/superscript $k$ used to distinguish the $k$ th-tier parameters in a multitier network.
} 
Corollary III.1: In mmWave cellular networks with truncated channel inversion power control and cutoff threshold $\rho_{o}$, the probability distribution function (PDF) of the transmit power of a typical user in the uplink is given by

$$
f_{P}(p)=\frac{\lambda(p) e^{-\Lambda(p)}}{\int_{0}^{P_{u}} \lambda(y) e^{-\Lambda(y)} \mathrm{d} y}, \quad 0 \leq p \leq P_{u}
$$

where

$$
\begin{aligned}
\lambda(p)= & \frac{2 \pi \lambda}{\alpha_{L} \rho_{O}^{2 / \alpha_{L}}} p^{\frac{2}{\alpha_{L}}-1} e^{-\beta\left(\frac{p}{\rho_{o}}\right)^{\frac{1}{\alpha_{L}}}} \\
& +\frac{2 \pi \lambda}{\alpha_{N} \rho_{O}^{2 / \alpha_{N}}} p^{\frac{2}{\alpha_{N}}-1}\left(1-e^{-\beta\left(\frac{p}{\rho_{o}}\right)^{\frac{1}{\alpha_{N}}}}\right)
\end{aligned}
$$

and

$$
\begin{aligned}
& \Lambda(p)=\frac{2 \pi \lambda}{\beta^{2}}\left(1-e^{-\beta\left(\frac{p}{\rho_{o}}\right)^{\frac{1}{\alpha_{L}}}}\left(1+\beta\left(\frac{p}{\rho_{o}}\right)^{\frac{1}{\alpha_{L}}}\right)\right) \\
& +\pi \lambda\left(\frac{p}{\rho_{o}}\right)^{\frac{2}{\alpha_{N}}}-\frac{2 \pi \lambda}{\beta^{2}}\left(1-e^{-\beta\left(\frac{p}{\rho_{o}}\right)^{\frac{1}{\alpha_{N}}}}\left(1+\beta\left(\frac{p}{\rho_{o}}\right)^{\frac{1}{\alpha_{N}}}\right)\right) .
\end{aligned}
$$

Proof: The proof follows directly from proof of the multitier mmWave cellular networks.

Note that $1-e^{-y}(1+y)$ is strictly increasing in $y$ for $y>0$, hence, the first term of $\Lambda(p)$ in (8) is greater than the third term and $\Lambda(p)$ is strictly positive for all BS density $\lambda$, blockage parameter $\beta$, cutoff threshold $\rho_{O}$, and pathloss exponent $\alpha_{N} \geq \alpha_{L}>0$. Consequently, increasing the cutoff threshold $\rho_{O}$ leads to increase in the truncation outage probability as long as $\alpha_{N} \geq \alpha_{L}$. In other words, the higher the cutoff threshold, the poorer the mmWave network performance in terms of the truncation outage. As we will show in the later section, a low cutoff threshold could actually deteriorate the mmWave network performance in terms of the SINR outage probability. Hence, it is essential to manage the tradeoff between SINR outage and truncation outage probabilities using the cutoff threshold. On another note, by expanding (8), it can be seen that increasing the blockage parameter value $\beta$ leads to a reduction in $\Lambda(p)$ for fixed mmWave BS density $\lambda$, cutoff threshold $\rho$, and pathloss exponent $\alpha_{N}>\alpha_{L}$. Thus, the truncation outage probability also increases with increasing blockage parameter $\beta$. Increasing the blockage parameter implies decreasing the average LOS range, and hence, we have more NLOS paths requiring a much higher transmit power to meet the receiver sensitivity requirement. Regarding the expectation of the user transmission power, i.e., the average user transmission power, it is not straightforward to gain insights. However, from (7), we expect the plot of the average transmit power to be characterized from the LOS-based average transmit power and the NLOS-based average transmit power. We validate this observation later in the numerical results section.

\section{B. SINR Outage Probability}

For an active typical user, the SINR at its connected BS in the $j$ th tier (termed as the reference mmWave BS) can be written as

$$
\operatorname{SINR}_{j}=\frac{\rho_{o}^{j}\left|g_{o}\right|^{2} \mathcal{G}_{j}}{\sigma^{2}+\sum_{k=1}^{K} \sum_{z \in \mathcal{Z}_{k}} P_{z}\left|g_{z}\right|^{2} G_{z}^{j} L\left(D_{z}\right)}
$$

where $\rho_{o}^{j}$ is the long-term average received signal power (normalized by $\mathcal{G}_{j}$ ) due to the truncated channel inversion power control, $\mathcal{Z}_{k}$ is the set of interfering users associated with a BS in the $k$ th tier, $L\left(D_{z}\right)$ is the pathloss from the interfering users to the reference BS, $\sigma^{2}$ is the noise power, and $G_{z}^{j}$ is the directivity gain on an interfering link. To help with the analytical tractability, we model the small-scale fading $g_{z}$ as a Nakagami random variable with parameter $N$ for both the LOS and NLOS [27]. ${ }^{2}$

The SINR outage probability $\mathcal{O}_{s}^{j}$ is the probability that the instantaneous SINR experienced at the reference mmWave BS in the $j$ th tier is less than the target $\operatorname{SINR} \theta_{j}$, i.e., $\mathcal{O}_{s}^{j}=\mathbb{P}\left(\operatorname{SINR}_{j}<\right.$ $\left.\theta_{j}\right) .{ }^{3}$ Given that the average received signal at any of the BSs in the $j$ th tier is equivalent to the cutoff threshold of the $j$ th tier represented by $\rho_{o}^{j}$, the SINR outage probability of the $j$ th tier can be expressed as

$$
\begin{aligned}
\mathbb{P} & \left(\operatorname{SINR}_{j} \leq \theta_{j}\right) \\
& =\mathbb{P}\left\{\rho_{o}^{j}\left|g_{o}\right|^{2} \mathcal{G}_{j} \leq \theta_{j}\left(\sigma^{2}+\sum_{k=1}^{K}\left(I_{L}^{k}+I_{N}^{k}\right)\right)\right\}
\end{aligned}
$$

where $I_{L}^{k}$ and $I_{N}^{k}$ are the aggregate interference from LOS and NLOS users of the $k$ th tier, respectively. Note that $I_{L}^{j}$ and $I_{N}^{j}$ represents the LOS and NLOS cotier interference, respectively, and $I_{L}^{k}$ and $I_{N}^{k} \forall k \neq j$ denotes the LOS and NLOS cross-tier interference, respectively. Noting that $\left|g_{o}\right|^{2}$ is normalized gamma random variable with parameter $N$, we have the following approximation:

$$
\begin{aligned}
& \mathbb{P}\left\{\left|g_{o}\right|^{2} \leq \theta_{j}\left(\sigma^{2}+\sum_{k=1}^{K} I_{L}^{k}+\sum_{k=1}^{K} I_{N}^{k}\right) /\left(\rho_{o}^{j} \mathcal{G}_{j}\right)\right\} \\
& \stackrel{(a)}{\approx} 1-\left(1-\mathbb{E}\left[\left(1-e^{-\frac{\eta \theta_{j}\left(\sigma^{2}+\sum_{k=1}^{K} I_{L}^{k}+\sum_{k=1}^{K} I_{N}^{k}\right)}{\rho_{o}^{j} \mathcal{G}_{j}}}\right)^{N}\right]\right) \\
& \stackrel{(b)}{=} 1-\sum_{n=1}^{N}(-1)^{n+1}\left(\begin{array}{c}
N \\
n
\end{array}\right) \mathbb{E}_{\Phi}\left[e^{-\frac{n \eta \theta_{j}\left(\sigma^{2}+\sum_{k=1}^{K} I_{L}^{k}+\sum_{k=1}^{K} I_{N}^{k}\right)}{\rho_{o}^{j} \mathcal{G}_{j}}}\right] \\
& =1-\sum_{n=1}^{N}(-1)^{n+1}\left(\begin{array}{c}
N \\
n
\end{array}\right) e^{-s n \sigma^{2}} \prod_{k=1}^{K} \mathcal{L}_{I_{L}^{k}}(s n) \prod_{k=1}^{K} \mathcal{L}_{I_{N}^{k}}(s n) \\
& =\sum_{n=0}^{N}(-1)^{n}\left(\begin{array}{c}
N \\
n
\end{array}\right) e^{-s n \sigma^{2}} \prod_{k=1}^{K} \mathcal{L}_{I_{L}^{k}}(s n) \prod_{k=1}^{K} \mathcal{L}_{I_{N}^{k}}(s n)
\end{aligned}
$$

where $s=\frac{\eta \theta_{j}}{\rho_{o}^{j} \mathcal{G}_{j}}$ and $\eta=N(N !)^{-\frac{1}{N}} .(a)$ follow from the fact that $\left|g_{0}\right|^{2}$ is a normalized gamma random variable with parameter $N$ and the fact that for a constant $\gamma>0$, the probability $\mathbb{P}\left(\left|g_{0}\right|^{2}<\gamma\right)$ is tightly upper bounded by $\left[1-\exp \left(-\gamma N(N !)^{-\frac{1}{N}}\right)\right]^{N}$ [28]. Furthermore, the expectation is with respect to the random variables $I_{L}^{k}$ and $I_{N}^{k}$, which are approximated to be independent. (b) follows from the binomial theorem and the assumption that $N$ is an integer, and $\mathcal{L}_{I_{L}^{k}}$ and $\mathcal{L}_{I_{N}^{k}}$ denote the Laplace transforms of the random variables $I_{L}^{k}$ and $I_{N}^{k}$, respectively.

As mentioned earlier, the location of the interfering users do not create a PPP as a result of the correlation among the users from the channel assignment process. The interfering users are thus better modeled by using soft-core processes, which can

\footnotetext{
${ }^{2}$ To maintain completeness, all the simulations utilize Nakagami random variable with parameter $N$ for LOS and Rayleigh fading for NLOS.

${ }^{3}$ We consider that each of the tiers has its own SINR threshold which is represented by $\theta_{k}$.
} 
capture such correlation [29]. However, most soft-core processes lack analytical tractability [30], [31], thus making the expression for the Laplace transforms of the aggregate LOS and NLOS interference, $\mathcal{L}_{I_{L}^{k}}$ and $\mathcal{L}_{I_{N}^{k}}$, respectively, unobtainable. Hence, we approximate the location of the interfering users with a PPP. Note that the approximation has been shown to be accurate for the UHF network when the correlation among the interfering nodes and the reference receiver is captured [15], [16]. Our model here also captures this correlation. The accuracy of our assumption will be verified later through simulations. Based on the PPP approximation, and the independent and identical distributed transmit power for the set of interfering users in the uplink channel, the SINR outage probability of a typical user in the $j$ th tier of mmWave cellular networks can be obtained from the following theorem.

Theorem III.2: In a $K$-tier mmWave network with truncated channel inversion power control where each tier is distinguished by its density $\lambda_{k}$, cutoff threshold $\rho_{o}^{k}$, blockage parameter $\beta_{k}$, antenna parameters, $G_{b k}^{\max }, G_{b k}^{\min }$, and $\zeta_{r k}$, LOS pathloss exponent $\alpha_{L}^{k}$ and NLOS pathloss exponent $\alpha_{N}^{k}$, the SINR outage probability of a typical user in the $j$ th tier is given by

$$
\mathcal{O}_{s}^{j}=\sum_{n=0}^{N}(-1)^{n}\left(\begin{array}{c}
N \\
n
\end{array}\right) \exp \left(-\frac{\eta n \theta_{j} \sigma^{2}}{\rho_{o}^{j} \mathcal{G}_{j}}-\sum_{k=1}^{K}\left(Q_{n}^{k}+V_{n}^{k}\right)\right)
$$

where

$$
\begin{aligned}
& Q_{n}^{k}=2 \pi \lambda_{k} \sum_{v=1}^{4} b_{v}^{j} q_{v}^{j \frac{2}{\alpha_{L}^{j}}} \\
& \times \int_{\mathcal{A}_{j}}^{\infty} \int_{0}^{P_{u}} \mathcal{F}\left(N, \frac{y^{-\alpha_{L}^{j}}}{N}\right) e^{-\beta_{j}\left(q_{v}^{j} p\right)^{\frac{1}{\alpha_{L}^{j}}} y} y p^{\frac{2}{\alpha_{L}^{j}}} f_{P_{k}}(p) \mathrm{d} p \mathrm{~d} y \\
& V_{n}^{k}=2 \pi \lambda_{k} \sum_{v=1}^{4} b_{v}^{j} q_{v}^{j} \frac{2}{\alpha_{N}^{j}} \times \int_{\mathcal{B}_{j}}^{\infty} \int_{0}^{P_{u}} \mathcal{F}\left(N, \frac{y^{-\alpha_{N}^{j}}}{N}\right) \\
& \times\left(1-e^{-\beta_{j}\left(q_{v}^{j} p\right)^{\frac{1}{\alpha_{N}^{j}}} y}\right) y p^{\frac{2}{\alpha^{j}}} f_{P_{k}}(p) \mathrm{d} p \mathrm{~d} y, \\
& \eta=N(N !)^{-\frac{1}{N}}, \mathcal{A}_{j}=\left(\frac{\eta n \theta_{j} a_{v}^{j} \rho_{o}^{k}}{\rho_{o}^{j} \mathcal{G}_{j}}\right)^{-\frac{1}{\alpha_{L}^{j}}}, \mathcal{B}_{j}=\left(\frac{\eta n \theta_{j} a_{v}^{j} \rho_{o}^{k}}{\rho_{o}^{j} \mathcal{G}_{j}}\right)^{-\frac{1}{\alpha_{N}^{j}}} \\
& \mathcal{F}(N, y)=1-\frac{1}{(1+y)^{N}}, q_{v}^{j}=\frac{\eta n \theta_{j} a_{v}^{j}}{\rho_{o}^{j} \mathcal{G}_{j}}, a_{v}^{j} \text { and } b_{v}^{j} \text { are the antenna }
\end{aligned}
$$
directivity parameters defined in Section II and $f_{P_{k}}(p)$ is defined in (1).

\section{Proof: See Appendix B ${ }^{4}$}

Though this approximates, the SINR outage probability, we find that the expression compares very well with the simulation results in Section V-A. Furthermore, the expression here captures the user maximum power constraint contrary to the prior result on the uplink of mmWave networks in [24], which is based on an unbounded power constraint. The maximum power constraint is very important in the uplink power control of mmWave network due to the significant difference in the LOS and NLOS pathloss exponent. We show the impact of the maximum power constraint later in Section $\mathrm{V}$. It has been shown in [8] that the LOS probability function can be approximated by a step function in a dense mmWave

\footnotetext{
${ }^{4}$ Note that the SINR outage probability for the single-tier mmWave can be obtained from Theorem III. 2 by setting $j=k=K=1$, and using the distribution of the transmit power of the typical user in a single-tier defined in Corollary III.1.
}

network. Hence, in the next section, we propose to simplify our uplink system model and the subsequent analysis by using a step function approximation of the LOS probability function.

\section{ANALYSIS OF THE UPLINK OF DENSE MMWAVE NETWORKS}

In this section, we present the analysis for the uplink of a dense mmWave cellular network. The motivation for the dense network analysis is based on the fact that mmWave cellular networks must be dense in order to achieve its forecasted gain [8]. Here, we approximate the LOS probability $\mathbb{P}\left(\operatorname{LOS}_{k}\right)$ by using a step function such that the LOS probability $\mathbb{P}\left(\operatorname{LOS}_{k}\right)$ is taken to be 1 when the link is within a circular disc $\mathcal{B}\left(0, R_{B}\right)$ centered at the reference mmWave BS and 0 when outside the disc. Next, we present uplink signal-to-interference-ratio (SIR) distribution for the dense multitier mmWave networks, which we later degrade to the single-tier scenario.

\section{A. Outage Analysis in Dense Multitier mmWave Networks}

The dense mmWave network will be interference limited with mainly LOS interferers limiting its performance. Hence, we ignore both the noise power and the NLOS interfering users in the analysis. Furthermore, according to [3], the signal power from LOS interferers are nearly deterministic, hence, we also ignore the small-scale fading. Consequently, the SIR at the BS that the typical user connects to in the $j$ th tier can be expressed from (9) as

$$
\operatorname{SIR}_{j}=\frac{\rho_{o}^{j} \mathcal{G}_{j}}{\sum_{k=1}^{K} \sum_{z \in \mathcal{Z}_{k} \cap \mathcal{B}\left(0, R_{B}\right)} P_{z} G_{z}^{j} L\left(D_{z}\right)} .
$$

As mentioned earlier, for the dense deployment, the LOS interferers are dominant and the SINR outage probability in the $j$ th tier can thus be approximated as

$$
\mathbb{P}\left(\operatorname{SIR}_{j} \leq \theta_{j}\right)=\mathbb{P}\left\{\rho_{o}^{j} \mathcal{G}_{j} \leq \theta_{j} \sum_{k=1}^{K} I_{L}^{k}\right\}
$$

where $I_{L}^{k}=\sum_{z \in \mathcal{Z}_{k} \cap \mathcal{B}\left(0, R_{B}\right)} P_{z} G_{z} L\left(D_{z}\right)$ is the interference power received at the reference $\mathrm{BS}$ from users connected to $\mathrm{BSs}$ in the $k$ th tier. Note that the average receive signal at the reference BS normalized by the directivity gain $\mathcal{G}_{j}$ is equivalent to the cutoff threshold $\rho_{o}^{j}$. The SINR outage probability can be approximated as

$$
\begin{aligned}
\mathbb{P}\left\{\rho_{o}^{j} \mathcal{G}_{j}\right. & \left.<\theta_{j} \sum_{k=1}^{K} I_{L}^{k}\right\} \stackrel{(a)}{\approx} \mathbb{P}\left\{h<\frac{\theta_{j} \sum_{k=1}^{K} I_{L}^{k}}{\rho_{o}^{j} \mathcal{G}_{j}}\right\} \\
& \stackrel{(b)}{=} 1-\left(1-\mathbb{E}_{\Phi_{L}}\left[\left(1-e^{-\frac{\eta \theta_{j} \sum_{k=1}^{K} I_{L}^{k}}{\rho_{o}^{j} \mathcal{G}_{j}}}\right)^{L}\right]\right) \\
& =1-\sum_{l=1}^{L}\left(\begin{array}{c}
L \\
l
\end{array}\right)(-1)^{l+1} \prod_{k=1}^{K} \mathcal{L}_{I_{L}^{k}}(s l) \\
& =\sum_{l=0}^{L}\left(\begin{array}{c}
L \\
l
\end{array}\right)(-1)^{l} \prod_{k=1}^{K} \mathcal{L}_{I_{L}^{k}}(s l)
\end{aligned}
$$

where the dummy variable $h$ in $(a)$ is used to denote normalized gamma variable with parameter $L$. Note that the distribution of the normalized gamma variable converges to an identity when its parameters tend to infinity, $(b)$ follows from [28] such that 
the probability $\mathbb{P}\left(|h|^{2}<\gamma\right)$ is tightly upper bounded by $[1-$ $\left.\exp \left(-\gamma N(N !)^{-\frac{1}{N}}\right)\right]^{N}$ and $s=\frac{\eta \theta_{j}}{\rho_{o}^{j} \mathcal{G}_{j}}$. In the following theorem, we summarize the main result for the SIR outage distribution in a multitier network.

Theorem IV.1: The SINR outage probability in the $j$ th tier of a $K$-tier mmWave cellular network with truncated channel inversion power control can be approximated as (17), shown at the bottom of this page, where $\lambda_{0}^{k}=\lambda_{k} \pi R_{B}^{2}, \Gamma(a, b)=$ $\int_{b}^{\infty} t^{a-1} e^{-t} \mathrm{~d} t$ is the upper incomplete gamma function, $a_{v}^{j}$ and $b_{v}^{j}$ are the antenna directivity parameters defined in Section II, $\eta=L(L !)^{-\frac{1}{L}}, L$ is used in the approximation and $f_{P_{k}}(p)$ is defined as

$$
f_{P_{k}}(p)=\frac{\sum_{c=1}^{K} \frac{2 \pi \lambda_{c} p^{\frac{2}{\alpha_{L}^{c}}-1}}{\alpha_{L}^{c}\left(\rho_{o}^{k}\right)^{\frac{2}{\alpha_{L}^{c}}}}}{1-e^{-\sum_{a=1}^{K} \pi \lambda_{a}\left(\frac{P_{u}}{\rho_{o}^{k}}\right)^{\frac{2}{\alpha_{L}^{a}}}}} e^{-\sum_{b=1}^{K} \pi \lambda_{b}\left(\frac{p}{\rho_{o}^{k}}\right)^{\frac{2}{\alpha_{L}^{b}}}} .
$$

Proof: See Appendix C.

For a single-tier dense mmWave network, the approximation of SINR outage probability can be obtained from the following corollary.

Corollary IV.1: The SINR outage probability in a single-tier mmWave cellular network with truncated channel inversion power control can be approximated as (18), shown at the bottom of this page, where $\lambda_{0}=\lambda \pi R_{B}^{2}, \Gamma(a, b)=\int_{b}^{\infty} t^{a-1} e^{-t} \mathrm{~d} t$ is the upper incomplete gamma function, $a_{v}$ and $b_{v}$ are the antenna directivity parameters defined in Section II, $\eta=L(L !)^{-\frac{1}{L}}, L$ is the number of terms used in the approximation and $f_{P}(p)$ is defined as

$$
f_{P}(p)=\frac{2 \pi \lambda p^{\frac{2}{\alpha_{L}}-1} e^{-\pi \lambda\left(\frac{p}{\rho_{o}}\right)^{\frac{2}{\alpha_{L}}}}}{\alpha_{L} \rho_{O}^{\frac{2}{\alpha_{L}}}\left(1-e^{-\pi \lambda\left(\frac{P_{u}}{\rho_{o}}\right)^{\frac{2}{\alpha_{L}}}}\right)} .
$$

Proof: The proof follows directly from the proof of the multitier mmWave cellular networks and is omitted here.

\section{NUMERICAL RESULTS}

In this section, we present numerical results to illustrate our analytical findings for both the single-tier and two-tier mmWave cellular networks. Unless otherwise stated, we set the BS densities $\lambda_{1}=10 \mathrm{BS} / \mathrm{km}^{2}$ and $\lambda_{2}=2 \lambda_{1}$, the maximum transmit power $P_{u}=1 \mathrm{~W}, \sigma^{2}=-110 \mathrm{dBm}$, the tier blockage parameters $\beta_{1}=0.0071$ and $\beta_{2}=0.0143$ with corresponding pathloss exponent $\alpha_{L}^{1}=2, \alpha_{N}^{1}=4, \alpha_{L}^{2}=2.9, \alpha_{N}^{2}=5$, and the

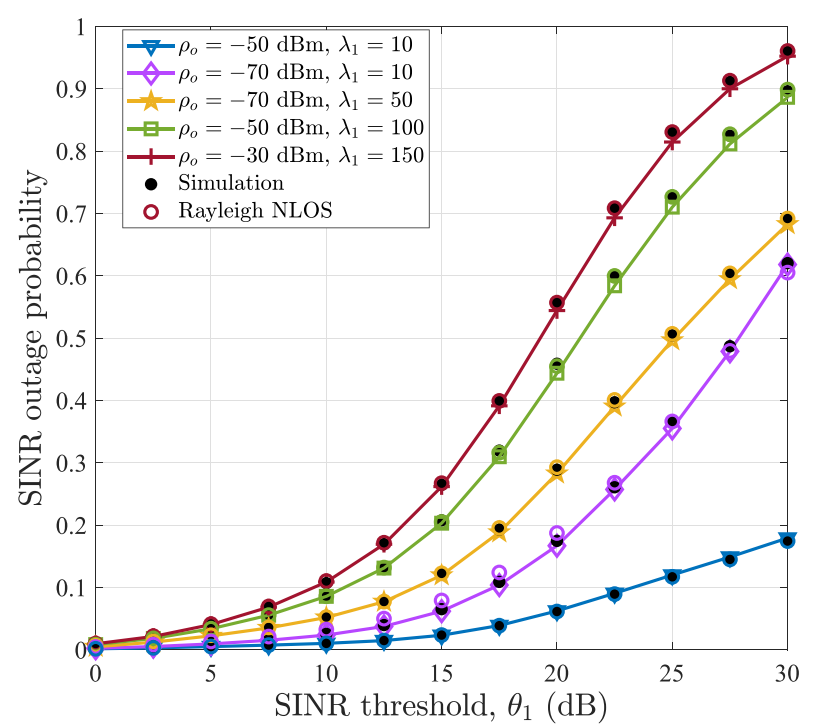

Fig. 1. Comparison of the analytical results with simulation in single-tier mmWave networks for $\beta_{1}=0.0071, \alpha_{L}^{1}=2$, and $\alpha_{N}^{1}=4$.

Nakagami fading parameter $N=3$. Furthermore, unless otherwise stated, the antenna parameters of the first and second tier BSs are equivalent such that $G_{b 1}^{\max }=G_{b 2}^{\max }=7 \mathrm{~dB}, G_{b 1}^{\min }=$ $G_{b 2}^{\max }=-10 \mathrm{~dB}$, and $\zeta_{b 1}=\zeta_{b 2}=30^{\circ}$, while that of the users are assumed to be characterized with $G_{u}^{\max }=7 \mathrm{~dB}, G_{u}^{\min }=$ $-10 \mathrm{~dB}$ and $\zeta_{u}=90^{\circ}$. In addition, we have utilized the system parameters of the first tier for the single-tier network results. Note that the simulation parameters used throughout this article are based on [8], [21], and [24].

\section{A. SINR Outage Probability}

In Figs. 1 and 2, we verify our derivation by plotting the analytical and simulation results for the single-tier and two-tier mmWave cellular networks, respectively. We also show the simulation results for the case where the NLOS is modeled with Rayleigh small-scale fading in Fig. 1. The results show that our derived analytical model accurately captures the SINR outage probability for both the single-tier and multitier mmWave cellular networks. Hence, our derived model finds great application in the mmWave multitier network where each tier can be identified via its BS density, blockage parameter, and corresponding LOS and NLOS pathloss exponents, receiver sensitivity, and the BS

$$
\begin{aligned}
\overline{\mathcal{O}}_{s}^{j}= & \sum_{l=0}^{L}(-1)^{l}\left(\begin{array}{l}
L \\
l
\end{array}\right) \prod_{k=1}^{K} \exp \left(\int _ { 0 } ^ { P u } \left(\pi \lambda_{k}\left(\frac{p}{\rho_{o}^{k}}\right)^{\frac{2}{\alpha_{L}^{j}}}-\lambda_{0}^{k}\right.\right. \\
& \left.\left.+\frac{2 \pi \lambda_{k}}{\alpha_{L}^{j}} \sum_{v=1}^{4}\left(\frac{\eta l \theta_{j} a_{v}^{j}}{\rho_{o}^{j} \mathcal{G}_{j}}\right)^{\frac{2}{\alpha_{L}^{j}}} b_{v}^{j} p^{\frac{2}{\alpha_{L}^{j}}}\left(\Gamma\left(\frac{-2}{\alpha_{L}^{j}}, \frac{\eta l \theta_{j} a_{v}^{j} p}{\rho_{o}^{j} \mathcal{G}_{j} R_{B}^{\alpha_{L}}}\right)-\Gamma\left(\frac{-2}{\alpha_{L}^{j}}, \frac{\eta l \theta_{j} a_{v}^{j} \rho_{o}^{k}}{\rho_{o}^{j} \mathcal{G}_{j}}\right)\right)\right) f_{P_{k}}(p) \mathrm{d} x\right) \\
\overline{\mathcal{O}}_{s}= & \sum_{l=0}^{L}(-1)^{l}\left(\begin{array}{l}
L \\
l
\end{array}\right) \exp \left(\int _ { 0 } ^ { P u } \left(\pi \lambda\left(\frac{p}{\rho_{o}}\right)^{\frac{2}{\alpha_{L}}}-\lambda_{0}\right.\right. \\
& \left.\left.+\frac{2 \pi \lambda}{\alpha_{L}} \sum_{v=1}^{4}\left(\frac{\eta l \theta a_{v}}{\rho_{o} \mathcal{G}}\right)^{\frac{2}{\alpha_{L}}} b_{v} p^{\frac{2}{\alpha_{L}}}\left(\Gamma\left(\frac{-2}{\alpha_{L}}, \frac{\eta l \theta a_{v} p}{\rho_{o} \mathcal{G} R_{B}^{\alpha_{L}}}\right)-\Gamma\left(\frac{-2}{\alpha_{L}}, \frac{\eta l \theta a_{v}}{\mathcal{G}}\right)\right)\right) f_{P}(p) \mathrm{d} p\right)
\end{aligned}
$$




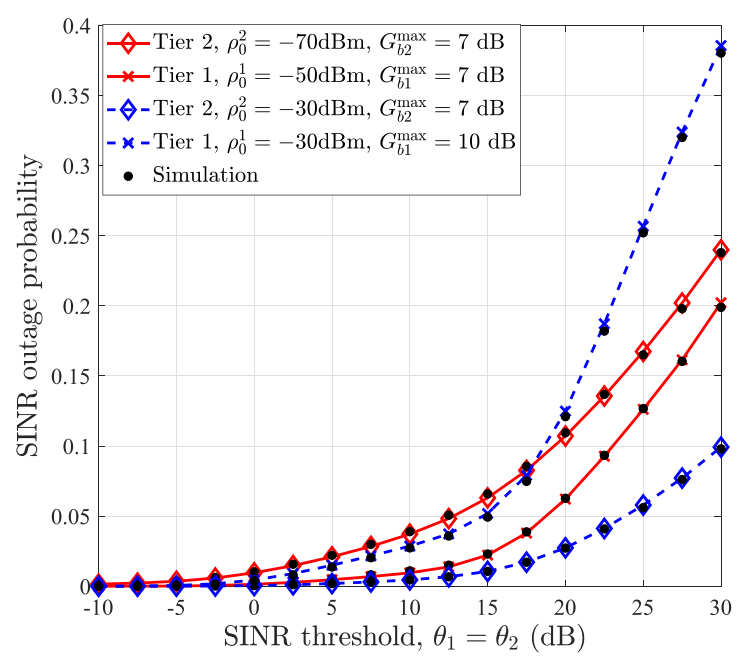

Fig. 2. Comparison of the analytical results with simulation in a two-tier mmWave network for $\beta_{1}=0.0071, \beta_{2}=0.0143, \alpha_{L}^{1}=2, \alpha_{N}^{1}=4, \alpha_{L}^{2}=$ 2.9 , and $\alpha_{N}^{2}=5$, and BS densities $\lambda_{1}=10 \mathrm{BS} / \mathrm{km}^{2}$ and $\lambda_{2}=2 \lambda_{1}$. Red and blue dashed-lines represent mmWave networks 1 and 2, respectively.

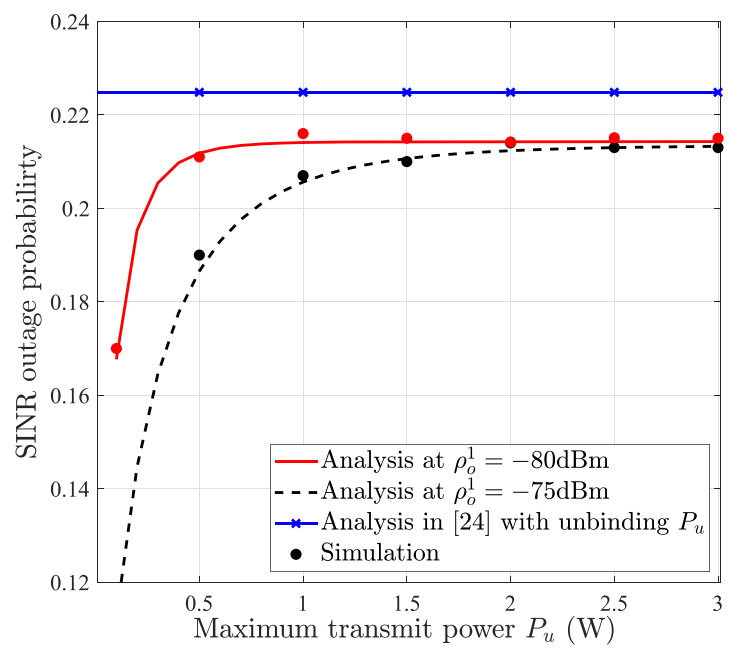

Fig. 3. Effect of the maximum transmit power in a single-tier mmWave network for $\theta_{1}=20 \mathrm{~dB}, \beta_{1}=0.0071, \alpha_{L}^{1}=2, \alpha_{N}^{1}=4$, and BS densities $\lambda=10 \mathrm{BS} / \mathrm{km}^{2}$.

antenna gain. Note that this validation is essential since the cumulative distribution of the SINR is based on the assumption that the active user constitute a PPP and that the transmit powers of the users are independent. Furthermore, independent LOS probability was assumed. The approach in this article, however, captures the correlation between the location of the reference mmWave BS and that of the interfering users. It also captures the correlation between the typical user's (served by the reference mmWave BS) transmit power and the interfering users' transmit powers. Furthermore, like [32], the results in Fig. 1 shows that modeling the NLOS links with Nakagami fading has minor impact on the SINR outage probability. However, note that all other simulation results assume Rayleigh small-scale fading for the NLOS links for completeness.

In Fig. 3, we compare our analysis with the one presented in [24], which does not incorporate the maximum user power constraint. Note that the analysis in [24] is for the single-tier, and hence, the comparison presented in Fig. 3 is also based on a single tier. As it can be seen, the maximum power constraint

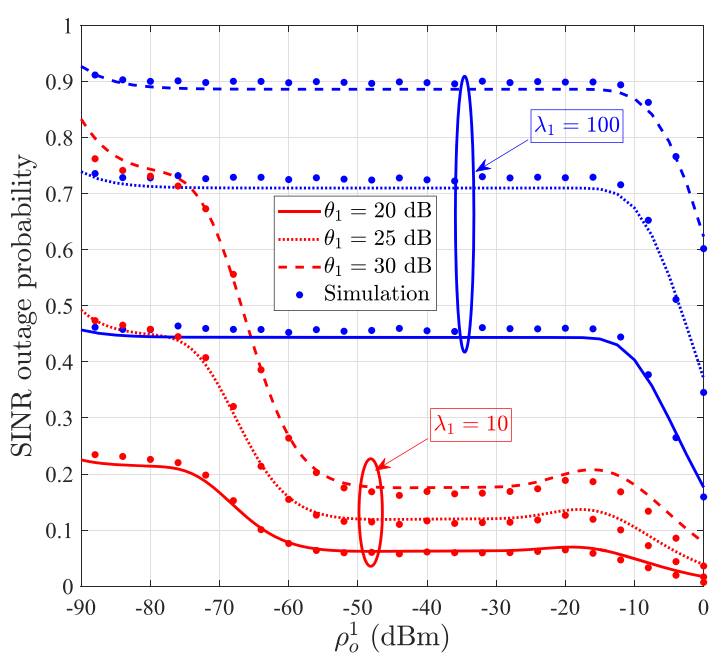

Fig. 4. Effect of the BS density on the SINR outage probability for $\lambda_{1}=$ 10 and $100 \mathrm{BS} / \mathrm{km}^{2}, \beta_{1}=0.0071, \alpha_{L}^{1}=2$, and $\alpha_{N}^{1}=4$ in a single-tier network.

significantly affects the SINR outage probability. The figure shows that the SINR outage probability derived in [24] does not vary with $P_{u}$, since the maximum power constraint is ignored in [24].

In Fig. 4, we plot the SINR outage probability for the uplink of a single-tier mmWave networks with truncated channel inversion power control for SINR threshold $\theta=20,25$, and $30 \mathrm{~dB}$, and BS densities $\lambda_{1}=10,100 \mathrm{BS} / \mathrm{km}^{2}$. It can be seen that the SINR outage probability of the mmWave deviates from that of the UHF network presented in [16]. More specifically, the following four sections can be identified from the plot for the BS density $\lambda_{1}=10 \mathrm{BS} / \mathrm{km}^{2}$.

1) A decrease in SINR outage probability can be seen for the cutoff threshold $\rho_{o}^{1}$ ranging from -100 to $-50 \mathrm{dBm}$ with a slow descent region observed for $\rho_{0}^{1}$ ranging from -85 to $-75 \mathrm{dBm}$.

2) A fairly stable outage probability can be observed for $\rho_{o}^{1}$ ranging from -50 to $-31 \mathrm{dBm}$.

3) An increase in SINR outage probability can be seen for $\rho_{o}^{1}$ ranging from -31 to $-18 \mathrm{dBm}$.

4) A decrease in SINR outage probability can be seen for $\rho_{o}^{1}$ ranging from -18 to $0 \mathrm{dBm}$.

This observation is a result of the large difference in the pathloss exponent of the LOS and NLOS propagation path, with each having its dominance region that also depends on the BS density and blockage parameter. The latter specifies the LOS range. Furthermore, the receiver sensitivity also specifies the density of active LOS and NLOS users and consequently, the interference received at the reference BS. It can also be observed from Fig. 4 that for the same SINR threshold, increasing the BS density leads to an increase in the SINR outage probability.

In Fig. 5, we show the effect of blockages on the SINR outage probability. Based on the LOS probability function $e^{-\beta_{1} r}$, a lower $\beta_{1}$ yields a larger number of LOS interfering user. Hence, the interference power increases when $\beta_{1}$ is lowered leading to a higher SINR outage probability for a lower $\beta_{1}$, as it can be seen in Fig. 5.

Figs. 6 and 7 shows the numerical results based on the dense network approximations in Section IV. In particular, Fig. 6 compares the dense network approximation of the SINR 


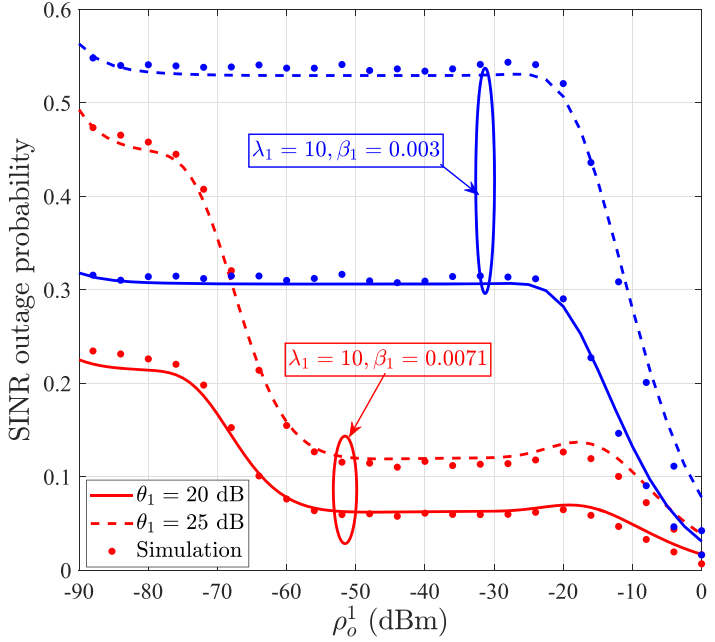

Fig. 5. Effect of the blockage parameter on the SINR outage probability for $\beta_{1}=0.0071$ and $0.003, \lambda_{1}=10 \mathrm{BS} / \mathrm{km}^{2}, \alpha_{L}^{1}=2$, and $\alpha_{N}^{1}=4$ in a singletier network.

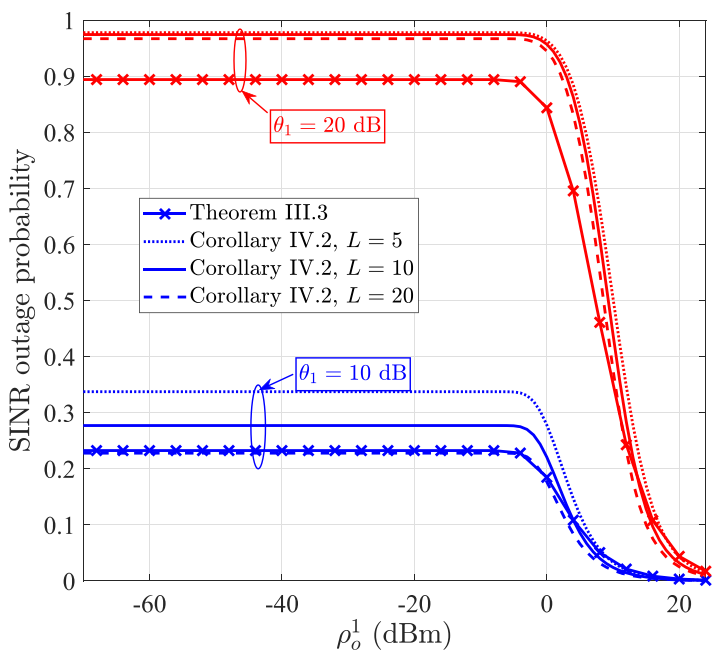

Fig. 6. Dense network approximation of the SINR outage probability in a single-tier mmWave network with $\alpha_{L}^{1}=2, \alpha_{N}^{1}=4, R_{B}=200 \mathrm{~m}$, and $\lambda_{0}=100$.

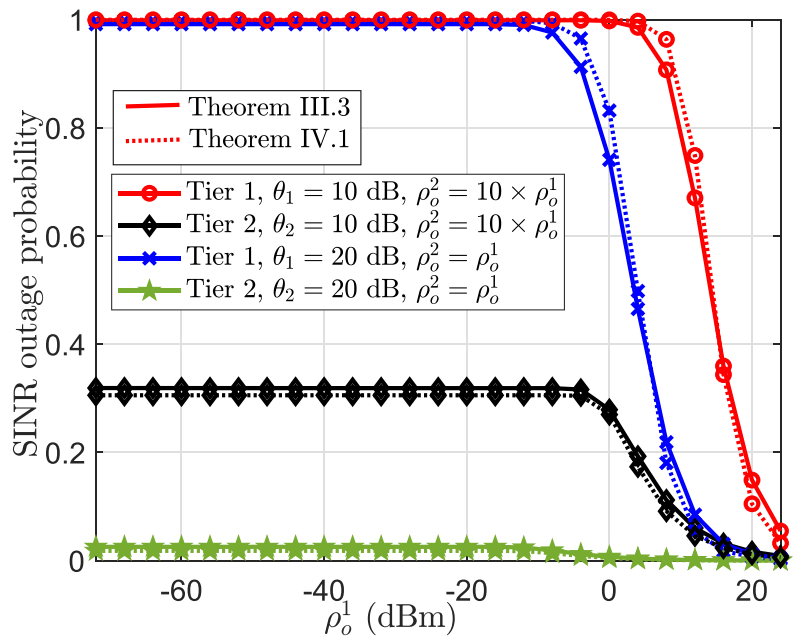

Fig. 7. Dense network approximation of the SINR outage probability in a two-tier mmWave network with $\alpha_{L}^{1}=2, \alpha_{N}^{1}=4, \alpha_{L}^{2}=2.9, \alpha_{N}^{2}=5, R_{B}=$ $200 \mathrm{~m}, \lambda_{0}=100, \lambda_{2}=2 \lambda_{1}, G_{b 1}^{\max }=10 \mathrm{~dB}$, and $G_{b 2}^{\max }=7 \mathrm{~dB}$.

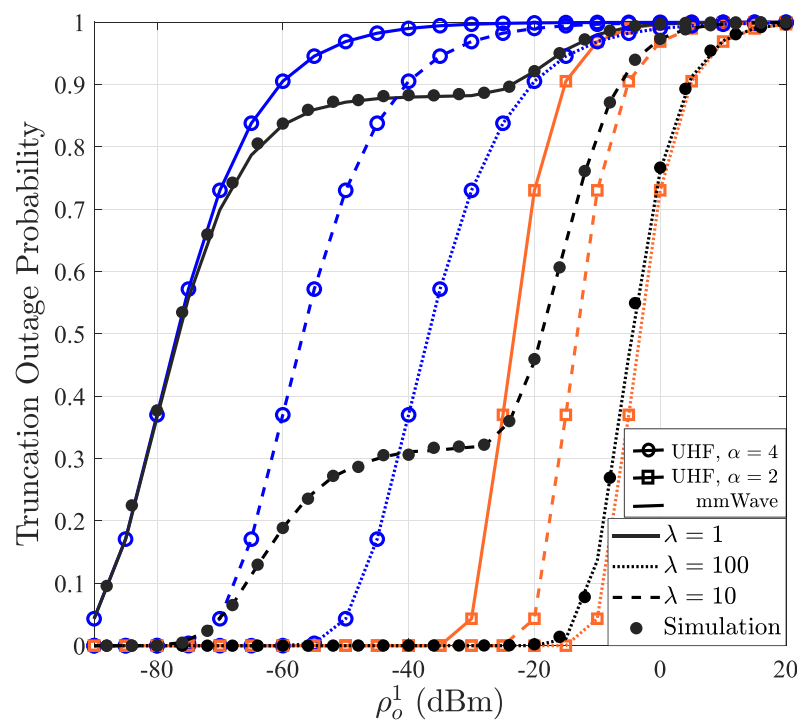

Fig. 8. Comparison of the truncation outage probability of mmWave and UHF cellular networks for BS density $\lambda=1,10$, and $100 \mathrm{BS} / \mathrm{km}^{2} . \beta_{1}=0.0071$, $\alpha_{L}^{1}=2$, and $\alpha_{N}^{1}=4$ in the mmWave network.

outage probability of a single-tier mmWave network given in Corollary IV.1 with the exact expression in Theorem III.2. For the dense network approximation, we take the radius of the LOS disk $R_{B}$ to be equal to $200 \mathrm{~m}$ and a relative BS density $\lambda_{0}=100$ where $\lambda_{0}=\lambda_{1} \pi R_{B}^{2}$. The dense network approximation becomes more accurate as $L$ increases. Furthermore, Fig. 7, compares the multitier dense network approximation in Theorem IV.1 with the exact expression in Theorem III.2, while focusing on a two-tier network. The first tier's BS density is obtained from $\lambda_{0}=\lambda_{1} \pi R_{B}^{2}$, while the second tier's BS density $\lambda_{2}=2 \lambda_{1}$ and $L$ equals 10 . It can be seen that similar to the single-tier network, the dense network approximation of the SINR outage probability is also fairly accurate for the multitier network.

\section{B. Truncation Outage Probability}

Fig. 8 compares the truncation outage probability for the uplink of mmWave and UHF cellular networks for BS density $\lambda=1,10$, and $100 \mathrm{BS} / \mathrm{km}^{2}$. The truncation outage probability of UHF networks has been defined in [16]. It can be seen that similar to the UHF case, increasing the cutoff threshold increases the outage probability since more users are unable to communicate due to insufficient transmit power. These results are in line with the insights previously in Section III-A. Furthermore, for BS densities $\lambda=1$ and 10 , the truncation outage of mmWave networks experience a slow growth region as the cutoff threshold increases before its saturation contrary to UHF networks, which does not experience a slow growth region. The slow growth region is due to the difference in the truncation outage probability for LOS and NLOS links at a given cutoff threshold. Meanwhile, for a high BS density of $\lambda=100$, the truncation outage probability of mmWave converges to that of UHF with $\alpha=2$ since more paths becomes LOS as the BS density increases. As expected, Fig. 8 shows that the truncation outage of mmWave networks reduces with as the BS density increases. This observation is due to the shortening of the average link lengths as the BS density is increased. 


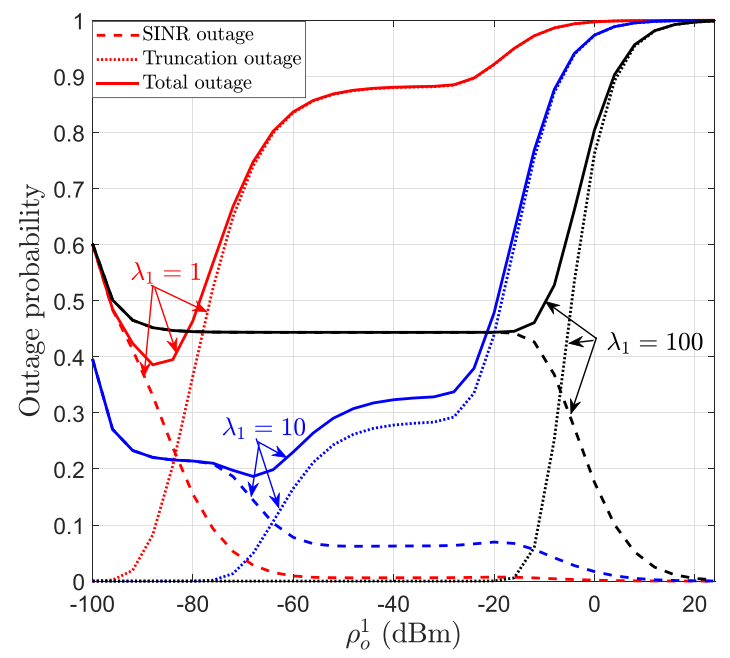

Fig. 9. Total outage probability in a single-tier mmWave network with $\theta_{1}=20 \mathrm{~dB}, \beta_{1}=0.0071, \alpha_{L}^{1}=2, \alpha_{N}^{1}=4$, and BS density $\lambda=1,10$, and $100 \mathrm{BS} / \mathrm{km}^{2}$.

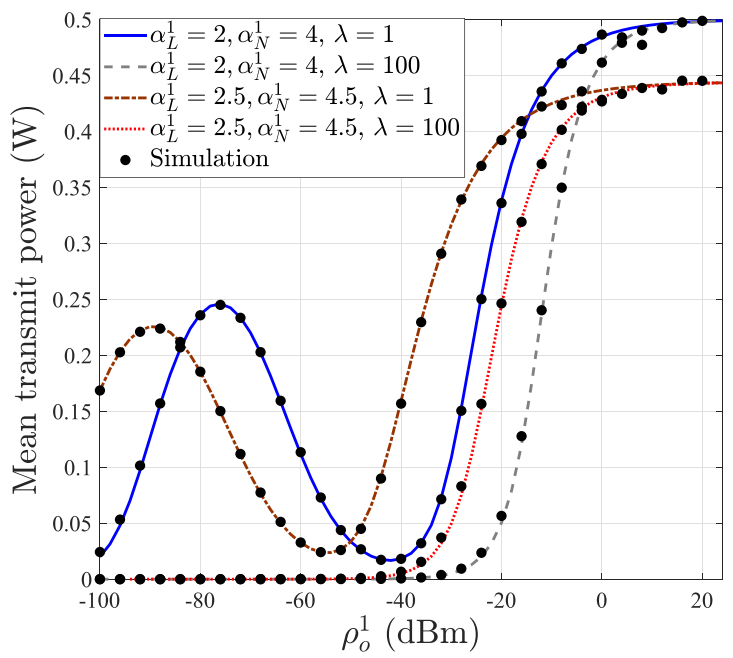

Fig. 10. Average transmit power in a single-tier mmWave network.

\section{Total Outage Probability}

In Fig. 9, we show the tradeoff introduced by the cutoff threshold $\rho_{o}^{1}$ on the total outage probability, which is defined as $\mathcal{O}_{t}=\mathcal{O}_{p}+\left(1-\mathcal{O}_{p}\right) \mathcal{O}_{s}$, for the single-tier mmWave network. It can be observed that $\rho_{o}^{1}$ tunes the tradeoff between the truncation and SINR outage probabilities and there exists a cutoff threshold $\rho_{o}^{1 \star}$ that minimizes the total outage probability in the single-tier network. Furthermore, the SINR probability dominates the total outage probability at lower cutoff threshold, while the truncation outage probability dominates the total outage probability at high values of the cutoff threshold $\rho_{o}^{1}$.

\section{Average User Transmit Power}

In Fig. 10, we plot the average transmit power of the users against the cutoff threshold $\rho_{0}^{1}$ for the single-tier mmWave network. It can be observed that for the case with BS density $\lambda=1$, the average transmit power increases with the cutoff threshold for $\rho_{o}^{1}$ ranging from -100 to $-75 \mathrm{dBm}$ and it then falls for $\rho_{o}^{1}$ ranging from -75 to $-40 \mathrm{dBm}$. Afterwards, average transmit power then rises with the cutoff threshold until its saturation. Note that increasing $\rho_{o}^{1}$, the user need to transmit a higher power to invert the pathloss and maintain a high threshold at the serving BS. However, each user is constrained to a maximum power $P_{u}$. Hence, a user becomes inactive when its transmit power requirement exceeds $P_{u}$. An initial increase in $\rho_{o}^{1}$ increases the transmit power of all users, and hence, the first increase in the mean transmit power. A point is reached where the density of active NLOS users starts to decrease with increasing cutoff threshold since the maximum power constraint cannot be satisfied, and hence, the reduction in the mean transmit power. The large discrepancy between the pathloss exponent of the LOS and NLOS users also means a large difference in the transmit power. However, a cutoff threshold is reached where the active LOS user starts to dominate since most NLOS users are inactive, thus leading to an increase in the mean transmit power till its saturation value given by $\lim _{\rho_{o}^{1} \rightarrow \infty} \mathbb{E}\left[P_{k}\right]=\frac{2}{\min \left(\alpha_{L}^{1}, \alpha_{N}^{1}\right)+2} P_{u}$. For dense deployment such as $\lambda=100$, most of the paths are LOS and the transmit power is nondecreasing with $\rho_{o}$ in this case.

\section{CONCLUSION}

In this article, we have presented a stochastic geometry-based framework to analyze the SINR outage probability in the uplink of multitier mmWave cellular networks with truncation channel inversion power control. The framework incorporates the effect of blockages, the per-user power control as well as the maximum power limitations of the users. Furthermore, each user controls its transmit power such that the received signal at its serving BS is equal to predefined cutoff threshold. Based on the proposed framework, we derived accurate expressions of the truncation outage probability and SINR outage probability for the uplink of the multitier mmWave cellular networks. Numerical results show that contrary to the conventional UHF networks, there exists a slow growth region for the truncated outage probability. Furthermore, increasing the cutoff threshold does not necessarily lead to a reduction in the SINR outage probability of the mmWave networks.

\section{APPENDIX}

\section{A. Proof of Theorem III.1}

Given that $y_{k}=\min _{m_{k} \in \Phi_{k}}\left(\left\|u-m_{k}\right\|^{\alpha_{s}^{k}}\right)$ is used to select the serving BS in the $k$ th tier. Then from [26], $f_{y_{k}}(y)=$ $\lambda_{k}(y) e^{-\Lambda_{k}(y)}$, where

$$
\begin{aligned}
\lambda_{k}(y)= & \frac{2 \pi \lambda_{k}}{\alpha_{L}^{k}} y^{\frac{2}{\alpha_{L}^{k}}-1} e^{-\beta_{k} y^{\frac{1}{\alpha_{L}^{k}}}} \\
& +\frac{2 \pi \lambda_{k}}{\alpha_{N}^{k}} y^{\frac{2}{\alpha_{N}^{k}}-1}\left(1-e^{-\beta_{k} y^{\frac{1}{\alpha_{N}^{k}}}}\right)
\end{aligned}
$$

and

$$
\begin{aligned}
\Lambda_{k}(y)= & \frac{2 \pi \lambda_{k}}{\beta_{k}^{2}}\left(1-e^{-\beta_{k} y^{\frac{1}{\alpha_{L}^{k}}}}\left(1+\beta_{k} y^{\frac{1}{\alpha_{L}^{k}}}\right)\right)+\pi \lambda_{k} y^{\frac{2}{\alpha_{N}^{k}}} \\
& -\frac{2 \pi \lambda_{k}}{\beta_{k}^{2}}\left(1-e^{-\beta_{k} y^{\frac{1}{\alpha_{N}^{k}}}}\left(1+\beta_{k} y^{\frac{1}{\alpha_{N}^{k}}}\right)\right)
\end{aligned}
$$


Noting that the user connects to the BS that provides the maximum average received signal. The typical user $u$ connects to the reference BS from the $j$ th tier, then $y_{j}=\min _{k}\left(y_{k}\right)$. The transmit power of the typical user connected to the reference user in the $j$ th tier is given by $P_{j}=\rho_{o}^{j} \min _{k}\left(y_{k}\right)$ where $P_{j} \leq P_{u}$. Consequently, the cumulative distribution function (CDF) of the transmit power can be expressed as

$$
F_{P_{j}}(p)=\frac{1-e^{-\sum_{k=1}^{K} \Lambda_{k}\left(\frac{p}{\rho_{o}^{j}}\right)}}{1-e^{-\sum_{k=1}^{K} \Lambda_{k}\left(\frac{P_{u}}{\rho_{o}^{J}}\right)}}
$$

and the PDF of the transmit power is given as

$$
\begin{aligned}
f_{P_{j}}(p) & =\frac{\mathrm{d} F_{P_{j}}(p)}{\mathrm{d} p} \\
& =\frac{\sum_{k=1}^{K} \bar{\lambda}_{k}(p)}{1-e^{-\sum_{a=1}^{K} \Lambda_{a}\left(\frac{P_{u}}{\rho_{o}^{j}}\right)}} e^{-\sum_{b=1}^{K} \Lambda_{b}\left(\frac{p}{\rho_{o}^{j}}\right)}
\end{aligned}
$$

where

$$
\begin{aligned}
\bar{\lambda}_{k}(p)= & \frac{2 \pi \lambda_{k}}{\alpha_{L}^{k} \rho_{o}^{j 2 / \alpha_{L}^{k}}} y^{\frac{2}{\alpha_{L}^{k}}-1} e^{-\beta_{k}\left(\frac{y}{\rho_{o}^{j}}\right)^{\frac{1}{\alpha_{L}^{k}}}} \\
& +\frac{2 \pi \lambda_{k}}{\alpha_{N}^{k} \rho_{o}^{j / \alpha_{N}^{k}}} y^{\frac{2}{\alpha_{N}^{k}}-1}\left(1-e^{-\beta_{k}\left(\frac{y}{\rho_{o}^{j}}\right)^{\frac{1}{\alpha_{N}^{k}}}}\right)
\end{aligned}
$$

and $\Lambda_{c}($.$) is given in (20).$

The PDF of $P_{j}$ has been normalized as a result of the truncated channel inversion power control. Furthermore, the $\eta$ th moment of $P_{j}$ is thus given by $\int_{0}^{P_{u}} p^{\eta} f_{P_{j}}(p)$ and the theorem is obtained.

\section{B. Proof of Theorem III.2}

Noting that the average interference received from any interfering user from the $k$ th tier is less than $\rho_{o}^{k}$. The sum interference received at the reference BS in the $j$ th tier from LOS users in the $k$ th can be expressed as

$$
I_{L}^{k}=\sum_{u_{z} \in \Phi_{L}^{k} \backslash\{o\}} \mathbf{1}\left(P_{z k}\left\|u_{z}\right\|^{-\alpha_{L}^{j}}<\rho_{o}^{k}\right) P_{z k} G_{z}^{j}\left|g_{z}\right|^{2}|| u_{z} \|^{-\alpha_{L}^{j}}
$$

where $\Phi_{L}^{k}$ is a PPP of LOS interfering users from the $k$ th tier. The indicator function is used to capture the correlation among the location of the interfering users and the location of the reference BS. Hence, the Laplace transform of the aggregate interference from LOS users in the $k$ th tier received by the reference mmWave BS in the $j$ th tier $\mathcal{L}_{I_{L}^{k}}$ can be computed as (25), shown at the bottom of this page, where (b1) follows from the independence of $\Phi_{L}^{k}, g_{z}, G_{z}^{j}$, and $P_{z k},(b 2)$ follows from the probability generation functional (PGFL) of the PPP [9] and the independence of the interference link directivity gain $G_{z}^{j}$ with probability distribution $a_{v}^{j}$ with probability $b_{v}^{j},(b 3)$ follows from computing the moment generating function of a gamma

$$
\begin{aligned}
& \mathcal{L}_{I_{L}^{k}}=\mathbb{E}_{\Phi_{L}^{k}}\left[e^{-s n I_{L}^{k}}\right]=\mathbb{E}_{\Phi_{L}^{k}}\left[e^{-s n \sum_{u z \in \Phi_{L}^{k} \backslash\{\rho\}} \mathbb{1}\left(P_{z k}\left\|u_{z}\right\|^{-\alpha_{L}^{j}}<\rho_{o}^{k}\right) P_{z k} G_{z}^{j}\left|g_{z}\right|^{2}\left\|u_{z}\right\|^{-\alpha_{L}^{j}}}\right] \\
& \stackrel{(b 1)}{=} \mathbb{E}_{\Phi_{L}^{k}}\left[\prod_{u_{z} \in \Phi_{L}^{k} \backslash\{0\}} e^{-s n \mathbb{\|}\left(P_{z k}\left\|u_{z}\right\|^{-\alpha_{L}^{j}}<\rho_{o}^{k}\right) P_{z k} G_{z}^{j}\left|g_{z}\right|^{2}\left\|u_{z}\right\|^{-\alpha_{L}^{j}}}\right]
\end{aligned}
$$

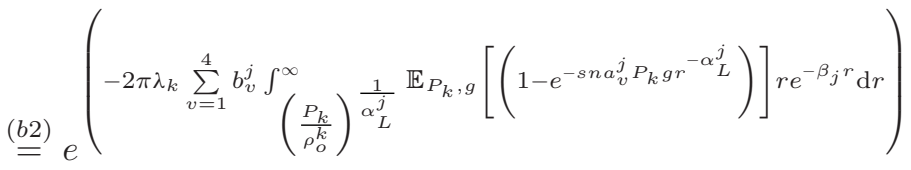$$
\stackrel{(b 3)}{=} e^{\left(-2 \pi \lambda_{k} \sum_{v=1}^{4} b_{v}^{j} \int^{\infty}\left(\frac{P_{k}}{\rho_{o}^{k}}\right)^{\frac{1}{\alpha_{L}^{j}}} \mathbb{E}_{P_{k}}\left[\left(1-\frac{1}{\left(1+\frac{s n a_{v}^{j} P_{k} r^{-\alpha_{L}^{j}}}{N}\right)^{N}}\right) r e^{-\beta_{j} r}\right] \mathrm{d} r\right)}
$$

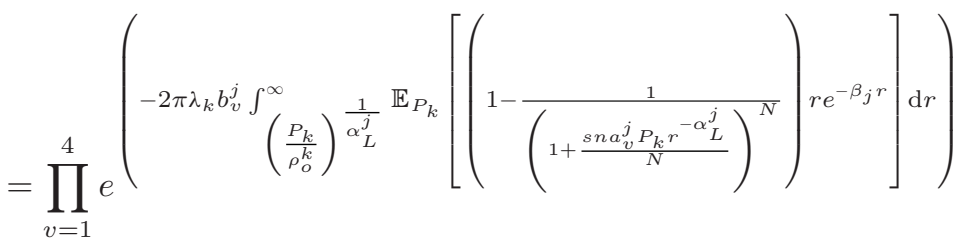$$
\stackrel{(b 4)}{=} \prod_{v=1}^{4} \exp \left(-2 \pi \lambda_{k} b_{v}^{j} q_{v}^{j \frac{2}{\alpha_{L}^{j}}} \int_{\left(s n a_{v}^{j} \rho_{o}^{k}\right)}^{\infty}-\frac{1}{\alpha_{L}^{j}} \int_{0}^{P_{u}}\left(1-\frac{1}{\left(1+\frac{y^{-\alpha_{L}^{j}}}{N}\right)^{N}}\right) \times y P_{k}^{\frac{2}{\alpha_{L}^{j}}} e^{-\beta_{j}\left(q_{v}^{j} P_{k}\right)^{\frac{1}{\alpha_{L}^{j}}} y} f_{P_{k}} \mathrm{~d} P \mathrm{~d} y\right)=e^{-Q_{n}^{k}}
$$ 


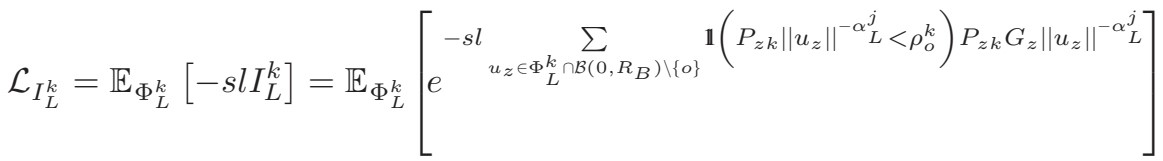

$$
\begin{aligned}
& \stackrel{(e 1)}{=} \exp \left(\mathbb{E}_{P_{k}}\left[\sum_{v=1}^{4}-2 \pi \lambda_{k} b_{v}^{j} \int_{\left(\frac{P_{k}}{\rho_{o}^{k}}\right)}^{R_{B}} \frac{1}{\alpha_{L}^{j}}\left(1-e^{s l P_{k} a_{v}^{j} r^{-\alpha_{L}^{j}}}\right) r \mathrm{~d} r\right]\right) \\
& \stackrel{(e 2)}{=} \exp \left(\mathbb{E}_{P_{k}}\left[\pi \lambda_{k}\left(\left(\frac{P_{k}}{\rho_{o}^{k}}\right)^{\frac{2}{\alpha_{L}^{j}}}-R_{B}^{2}\right)+\sum_{v=1}^{4} \frac{2 \pi \lambda_{k} b_{v}^{j}}{\alpha_{L}^{j}}\left(\operatorname{sla} a_{L}^{j}\right)^{\frac{2}{\alpha_{L}^{j}}} P_{k}^{\frac{2}{\alpha_{L}^{j}}} \int_{s l a_{v}^{j} P_{k} R_{B}^{-\alpha_{L}^{j}}}^{s l a_{v}^{j} \rho_{o}^{k}} \frac{e^{-w}}{1+\frac{2}{\alpha_{L}^{j}}} \mathrm{~d} v\right]\right) \\
& \stackrel{(e 3)}{=} \exp \left(\int_{0}^{P_{u}}\left(\pi \lambda_{k}\left(\left(\frac{P_{k}}{\rho_{o}^{k}}\right)^{\frac{2}{\alpha_{L}^{j}}}-R_{B}^{2}\right)+\sum_{v=1}^{4} \frac{2 \pi \lambda_{k} b_{v}^{j}}{\alpha_{L}^{j}}\left(\operatorname{sla} a_{L}^{j}\right)^{\frac{2}{\alpha_{L}^{j}}} P_{k}^{\frac{2}{\alpha_{L}^{j}}}\left(\Gamma\left(\frac{-2}{\alpha_{L}^{j}}, \frac{\eta l \theta_{j} a_{v}^{j} P_{k}}{\rho_{o}^{j} \mathcal{G}_{j} R_{B}^{\alpha_{L}^{j}}}\right)-\Gamma\left(\frac{-2}{\alpha_{L}^{j}}, \frac{\eta l \theta_{j} a_{v}^{j}}{\mathcal{G}_{j}}\right)\right)\right) f_{P_{k}} \mathrm{~d} P_{k}\right)
\end{aligned}
$$

random variable $g,(b 4)$ is obtained by changing the variables $y=r /\left(\operatorname{sna} a_{v}^{j} P_{k}\right)^{\frac{1}{\alpha_{L}^{j}}}$, while $f_{P_{k}}$ is given in (1). Furthermore, $q_{v}^{j}=s n a_{v}^{j}$.

Similarly, the Laplace transform of the aggregate interference from NLOS users in the $k$ th tier received by the reference BS in the $j$ th tier $\mathcal{L}_{I_{N}^{k}}$ can be expressed as

$$
\begin{aligned}
& \mathcal{L}_{I_{N}^{k}}=\mathbb{E}_{\Phi_{N}^{k}}\left[e^{-s n I_{N}^{k}}\right]=\prod_{v=1}^{4} \exp \\
& \times\left(-2 \pi \lambda_{k} b_{v}^{j} q_{v}^{j \frac{2}{\alpha^{j}}} \int_{\left(s n a_{v}^{j} \rho_{o}^{k}\right)}^{\infty}{ }^{-\frac{1}{\alpha_{N}^{j}}} \int_{0}^{P_{u}}\left(1-\frac{1}{\left(1+\frac{y^{-\alpha_{N}^{j}}}{N}\right)^{N}}\right)\right. \\
& \left.\times y P_{k}^{\frac{2}{\alpha_{N}^{j}}}\left(1-e^{-\beta_{k}\left(q_{v}^{j} P_{k}\right)^{\frac{1}{\alpha_{N}^{j}}} y}\right) f_{P_{k}} \mathrm{~d} P \mathrm{~d} y\right)=e^{-V_{n}^{k}} .
\end{aligned}
$$

\section{Proof of Theorem IV.1}

The proof is based on the key assumptions in Appendix B such that, where $(e 1)$ follows from computing the PGFL of the PPP $\Phi_{L}^{k},(e 2)$ follow from a change of variable $w=s l a_{v}^{j} P_{k} r^{-\alpha_{L}^{j}}$, and $(e 3)$ follows from the simplified PDF of the transmit power over the LOS region. The PDF of the uplink transmit power in the $k$ th tier of dense mmWave networks can be obtained by noting that $P_{k}=\rho_{o}^{k} r_{k}^{\alpha_{L}}$, where $r_{k}$ is the $k$ th tier uplink distance in the dense deployment, which follows a Rayleigh distribution $f_{r_{k}}(r)=2 \pi \lambda r e^{-\pi \lambda r^{2}}, 0 \leq r \leq \infty$. Consequently, following the same approach in Appendix A, we obtain the PDF of the $k$ th tier transmit power as

$$
f_{P_{j}}(x)=\frac{\sum_{k=1}^{K} \frac{2 \pi \lambda_{k} p^{\frac{2}{\alpha_{L}^{k}}-1}}{\alpha_{L}^{k}\left(\rho_{o}^{j}\right)^{\frac{2}{\alpha_{L}^{k}}}}}{1-e^{-\sum_{a=1}^{K} \pi \lambda_{a}\left(\frac{P_{u}}{\rho_{o}^{j}}\right)^{\frac{2}{\alpha_{L}^{a}}}}} e^{-\sum_{b=1}^{K} \pi \lambda_{b}\left(\frac{p}{\rho_{o}^{j}}\right)^{\frac{2}{\alpha_{L}^{b}}}} .
$$

Finally, the approximation of the SINR outage probability in the uplink of a multitier mmWave network given in (17) can be obtained by substituting (27), shown at the top of this page, for (14) and with $\lambda_{0}=\lambda \pi R_{B}^{2}$.

\section{REFERENCES}

[1] J. Andrews et al., "What will 5G be?" IEEE J. Sel. Areas Commun., vol. 32, no. 6, pp. 1065-1082, Jun. 2014

[2] Z. Pi and F. Khan, "An introduction to millimeter-wave mobile broadband systems," IEEE Commun. Mag., vol. 49, no. 6, pp. 101-107, Jun. 2011.

[3] T. Rappaport et al., "Millimeter wave mobile communications for 5G cellular: It will work!" IEEE Access, vol. 1, pp. 335-349, 2013.

[4] T. Rappaport, F. Gutierrez, E. Ben-Dor, J. Murdock, Y. Qiao, and J. Tamir, "Broadband millimeter-wave propagation measurements and models using adaptive-beam antennas for outdoor urban cellular communications," IEEE Trans. Antennas Propag., vol. 61, no. 4, pp. 1850-1859, Apr. 2013.

[5] S. Rangan, T. Rappaport, and E. Erkip, "Millimeter-wave cellular wireless networks: Potentials and challenges," Proc. IEEE, vol. 102, no. 3, pp. 366385, Mar. 2014.

[6] M. Akdeniz et al., "Millimeter wave channel modeling and cellular capacity evaluation," IEEE J. Sel. Areas Commun., vol. 32, no. 6, pp. 1164-1179, Jun. 2014.

[7] W. Roh et al., "Millimeter-wave beamforming as an enabling technology for 5G cellular communications: Theoretical feasibility and prototype results," IEEE Commun. Mag., vol. 52, no. 2, pp. 106-113, Feb. 2014.

[8] T. Bai and R. Heath, "Coverage and rate analysis for millimeter-wave cellular networks," IEEE Trans. Wireless Commun., vol. 14, no. 2, pp. 11001114, Feb. 2015.

[9] J. Andrews, F. Baccelli, and R. Ganti, "A tractable approach to coverage and rate in cellular networks," IEEE Trans. Commun., vol. 59, no. 11, pp. 3122-3134, Nov. 2011.

[10] X. Zhang and J. Andrews, "Downlink cellular network analysis with multislope path loss models," IEEE Trans. Commun., vol. 63, no. 5, pp. 18811894, May 2015.

[11] M. Ding, D. López-Pérez, G. Mao, P. Wang, and Z. Lin, "Will the area spectral efficiency monotonically grow as small cells go dense?" in Proc. IEEE Globecom, San Diego, CA, USA, Dec. 2015, pp. 1-7.

[12] H.-S. Jo, Y. J. Sang, P. Xia, and J. Andrews, "Heterogeneous cellular networks with flexible cell association: A comprehensive downlink SINR analysis," IEEE Trans. Wireless Commun., vol. 11, no. 10, pp. 3484-3495, Oct. 2012.

[13] S. Singh, H. S. Dhillon, and J. G. Andrews, "Offloading in heterogeneous networks: Modeling, analysis, and design insights," IEEE Trans. Wireless Commun., vol. 12, no. 5, pp. 2484-2497, May 2013.

[14] M. Di Renzo, A. Guidotti, and G. Corazza, "Average rate of downlink heterogeneous cellular networks over generalized fading channels: A stochastic geometry approach," IEEE Trans. Commun., vol. 61, no. 7, pp. 3050-3071, Jul. 2013. 
[15] T. Novlan, H. Dhillon, and J. Andrews, "Analytical modeling of uplink cellular networks," IEEE Trans. Wireless Commun., vol. 12, no. 6, pp. 2669-2679, Jun. 2013.

[16] H. ElSawy and E. Hossain, "On stochastic geometry modeling of cellular uplink transmission with truncated channel inversion power control," IEEE Trans. Wireless Commun., vol. 13, no. 8, pp. 4454-4469, Aug. 2014.

[17] J. Zhang, L. Xiang, D. W. K. Ng, M. Jo, and M. Chen, "Energy efficiency evaluation of multi-tier cellular uplink transmission under maximum power constraint," IEEE Trans Wireless Commun., vol. 16, no. 11, pp. 7092-7107, Nov. 2017.

[18] O. Onireti, A. Imran, M. Imran, and R. Tafazolli, "Energy efficient interfrequency small cell discovery in heterogeneous networks," IEEE Trans. Veh. Technol., vol. 65, no. 9, pp. 7122-7135, Sep. 2016.

[19] J. Andrews, A. K. Gupta, and H. S. Dhillon, "A primer on cellular network analysis using stochastic geometry," 2016, [Online]. Available: http://arxiv.org/abs/1604.03183

[20] M. D. Renzo, "Stochastic geometry modeling and analysis of multi-tier millimeter wave cellular networks," IEEE Trans. Wireless Commun., vol. 14 , no. 9, pp. 5038-5057, Sep. 2015.

[21] D. Maamari, N. Devroye, and D. Tuninetti, "Coverage in mmwave cellular networks with base station co-operation," IEEE Trans Wireless Commun., vol. 15, no. 4, pp. 2981-2994, Apr. 2016.

[22] C. Fang, B. Makki, and T. Svensson, "Coverage analysis for millimeter wave uplink cellular networks with partial zero-forcing receivers," in Proc. Int. Symp. Model. Optim. Mobile, Ad Hoc, Wireless Netw., May 2017, pp. 1-6.

[23] O. Onireti, A. Imran, and M. Imran, "Coverage analysis in the uplink of mmwave cellular network," in Proc. Eur. Conf. Netw. Commun., Jun. 2017, pp. 1-6.

[24] O. Onireti, A. Imran, and M. A. Imran, "Coverage, capacity and energy efficiency analysis in the uplink of mmwave cellular networks," IEEE Trans. Veh. Technol., vol. 67, no. 5, pp. 3982-3997, May 2018

[25] T. Bai, R. Vaze, and R. Heath, "Analysis of blockage effects on urban cellular networks," IEEE Trans. Wireless Commun., vol. 13, no. 9, pp. 5070-5083, Sep. 2014

[26] O. Onireti and M. A. Imran, "Truncated channel inversion power control for the uplink of mmWave cellular networks," in Proc. IEEE Sensor Array Multichannel Signal Process. Workshop, Jul. 2018, pp. 75-79.

[27] A. Thornburg, T. Bai, and R. W. Heath, "Performance analysis of outdoor mmwave ad hoc networks," IEEE Trans. Signal Process., vol. 64, no. 15, pp. 4065-4079, Aug. 2016.

[28] H. Alzer, "On some inequalities for the incomplete Gamma function," Math. Comput., vol. 66, no. 218, pp. 771-778, Apr. 1997.

[29] A. Guo and M. Haenggi, "Spatial stochastic models and metrics for the structure of base stations in cellular networks," IEEE Trans. Wireless Commun., vol. 12, no. 11, pp. 5800-5812, Nov. 2013.

[30] M. Haenggi, Stochastic Geometry for Wireless Networks. Cambridge, U.K.: Cambridge Univ. Press, 2013.

[31] H. ElSawy, E. Hossain, and M. Haenggi, "Stochastic geometry for modeling, analysis, and design of multi-tier and cognitive cellular wireless networks: A survey," IEEE Commun. Surv. Tut., vol. 15, no. 3, pp. 9961019, Jul. 2013

[32] X. Yu, J. Zhang, M. Haenggi, and K. B. Letaief, "Coverage analysis for millimeter wave networks: The impact of directional antenna arrays," IEEE J. Sel. Areas Commun., vol. 35, no. 7, pp. 1498-1512, Jul. 2017.

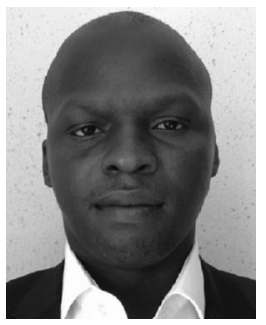

Oluwakayode Onireti (Member, IEEE) received the B.Eng. (Hons.) degree in electrical engineering from the University of Ilorin, Ilorin, Nigeria, in 2005, the M.Sc. (Hons.) degree in mobile and satellite communications, and the Ph.D. degree in electronics engineering from the University of Surrey, Guildford, U.K., in 2009 and 2012, respectively.

$\mathrm{He}$ is currently a Lecturer with the University of Glasgow, Glasgow, U.K. He has been actively involved in projects such as ROCKET, EARTH, Greencom, QSON, and Energy proportional EnodeB for LTE-Advanced, and Beyond. His main research interests include self-organizing cellular networks, millimeter wave communications, energy efficiency, wireless blockchain networks, multiple-input-multiple-output, and cooperative communications.

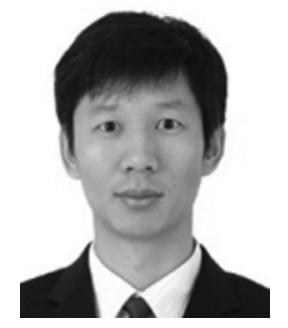

Lei Zhang (Member, IEEE) received the Ph.D. degree in array signal processing from the University of Sheffield, Sheffield, U.K., in 2011

$\mathrm{He}$ is a Lecturer with the University of Glasgow, Glasgow, U.K. He worked as a Research Engineer with Huawei Communication Technology Laboratory, and a Research Fellow with the 5G Innovation Centre, Institute of Communications, University of Surrey, Guildford, U.K. where he also holds a Visiting Position. His research interests include the communications and networks, including wireless blockchain networks, radio access network slicing, new air interface design, Internet of Things (IoT), multiantenna signal processing, massive multiple-input-multipleoutput systems, etc. He has 19 US/UK/EU/China granted/filed patents on wireless communications and authored and coauthored more than 100 peer-reviewed papers.

Dr. Zhang is an Associate Editor for the IEEE ACCESS and a Senior Member of the IEEE.

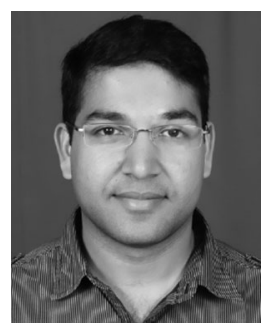

Ali Imran (Senior Member, IEEE) received the M.Sc. degree (with distinction) in mobile and satellite communications and the Ph.D. degree from the University of Surrey, Guildford, U.K., in 2007 and 2011, respectively. He is the Founding Director of AI4Networks Research Center, University of Oklahoma, Norman, OK, USA, where he is leading several multinational and industry lead projects on artificial intelligence (AI) for wireless networks. He is also a co-founder of a start-up AISON. Before joining University of Oklahoma (OU) in January 2014, for three years he has worked as a Research Scientist with QMIC, Doha, Qatar. Between October 2007 and October 2011, he has worked with the Institute of Communications Systems, University of Surrey, Guildford, U.K. In that position, he has contributed to a number of pan-European and international research projects while working in close collaboration with key industrial players. He has authored and coauthored more than 100 refereed journal and conference papers and has several patents granted and pending.

Dr. Imran is an Associate Fellow of Higher Education Academy (AFHEA), U.K.; the President of ComSoc Tulsa Chapter, a Member of Advisory Board for Special Technical Community on Big Data at the IEEE Computer Society; and a Board Member of ITERA. In 2018, he has been named William H. Barkow Presidential Professor at the University of Oklahoma. His research on AI-enabled wireless networks has played seminal role in this emerging area and has been supported by $\$ 4 \mathrm{M}$ in nationally and internationally competitive research funding and recognized by several prestigious awards such as the IEEE Green information and Communication Technology (ICT) Young Professional (YP) award 2017, the Vice President for Research and Partnerships (VPRP) Outstanding International Impact Award at the University of Oklahoma in 2017 and the Best Paper Award IEEE Computer Aided Modeling and Design of Communication Links and Networks (CAMAD) 2013. He is routinely invited to serve as an Advisor to key stakeholder in cellular network ecosystem and as a Speaker and a Panelist on international industrial fora and academic conferences on this topic.

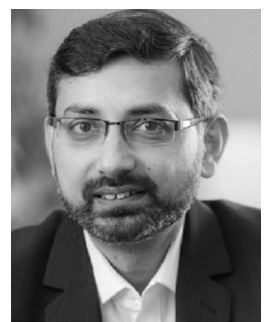

Muhammad Ali Imran (Senior Member, IEEE) received the M.Sc. (Distinction) and Ph.D. degrees from Imperial College London, U.K., in 2002 and 2007, respectively.

$\mathrm{He}$ is a Professor of wireless communication systems. He heads the Communications, Sensing and Imaging (CSI) research group, University of Glasgow, Glasgow, U.K., and is the Dean with the University of Glasgow, for the University of Electronic Science and Technology China programs. He is an Affiliate Professor with the University of Oklahoma, Norman, OK, USA, and a Visiting Professor with 5G Innovation Centre, University of Surrey, Guildford, U.K. He has more than 20 years of combined academic and industry experience with several leading roles in multimillion pounds funded projects. He has filed 15 patents; has authored/coauthored more than 400 journal and conference publications; was the Editor of 5 books and author of more than 20 book chapters; and has successfully supervised more than 40 postgraduate students at Doctoral level. He has been a Consultant to international projects and local companies in the area of self-organized networks. He has been interviewed by BBC, Scottish television, and many radio channels on the topic of $5 \mathrm{G}$ technology. His research interests include self-organized networks, wireless networked control systems, and the wireless sensor systems.

Prof. Imran is a Fellow of the Institution of Engineering and Technology and a Senior Fellow of the Higher Education Academy (HEA). 